\title{
Generalized arithmetic operators and their relationship to t-norms in interval-valued fuzzy set theory
}

\author{
Glad Deschrijver \\ Fuzziness and Uncertainty Modelling Research Unit \\ Department of Applied Mathematics and Computer Science \\ Ghent University, Krijgslaan 281 (S9), B-9000 Gent, Belgium
}

\begin{abstract}
In this paper we study extensions of the arithmetic operators $+,-, \cdot, \div$ to the lattice $\mathcal{L}^{I}$ of closed subintervals of the unit interval. Starting from a minimal set of axioms that these operators must fulfill, we investigate which properties they satisfy. We also investigate some classes of t-norms on $\mathcal{L}^{I}$ which can be generated using these operators; these classes provide natural extensions of the Lukasiewicz, product, Frank, Schweizer-Sklar and Yager t-norms to $\mathcal{L}^{I}$.
\end{abstract}

Key words: interval-valued fuzzy set, Łukasiewicz t-norm, product t-norm, Frank t-norm, Schweizer-Sklar t-norm, Yager t-norm, arithmetic operators

\section{Introduction}

Interval-valued fuzzy set theory $[15,19]$ is an extension of fuzzy theory in which to each element of the universe a closed subinterval of the unit interval is assigned which approximates the unknown membership degree. Another extension of fuzzy set theory is intuitionistic fuzzy set theory introduced by Atanassov [1]. In [9] it is shown that Atanassov's intuitionistic fuzzy set theory is equivalent to interval-valued fuzzy set theory and that both are equivalent to $L$-fuzzy set theory in the sense of Goguen [14] w.r.t. a special lattice $\mathcal{L}^{I}$.

In [5] we introduced additive and multiplicative generators on $\mathcal{L}^{I}$ based on a special kind of addition introduced in [6]. It was shown that the only t-norms

Email address: Glad.Deschrijver@UGent.be (Glad Deschrijver). 
on $\mathcal{L}^{I}$ which have a continuous additive generator on $\mathcal{L}^{I}$ that is a natural extension of an additive generator on the unit interval are pseudo-t-representable, and this is due to the typical construction of the addition operator introduced in [6]. Because of this limitation, in [11] another addition was introduced. In fact many more additions can be introduced. Therefore, in this paper we will study arithmetic operators on $\mathcal{L}^{I}$ in an axiomatic way. Starting from a minimal set of axioms that these operators must fulfill, we investigate which properties can be deduced. We will investigate some classes of t-norms and t-conorms which can be generated using the arithmetic operators on $\mathcal{L}^{I}$ and which are natural extensions of some well-known t-norms and t-conorms on the unit interval. In a future paper we will use the arithmetic operators to define additive and multiplicative generators on $\mathcal{L}^{I}$ in a more general way.

\section{The lattice $\mathcal{L}^{I}$}

Definition 1 We define $\mathcal{L}^{I}=\left(L^{I}, \leq_{L^{I}}\right)$, where

$$
\begin{aligned}
& L^{I}=\left\{\left[x_{1}, x_{2}\right] \mid\left(x_{1}, x_{2}\right) \in[0,1]^{2} \text { and } x_{1} \leq x_{2}\right\}, \\
& {\left[x_{1}, x_{2}\right] \leq_{L^{I}}\left[y_{1}, y_{2}\right] \Longleftrightarrow\left(x_{1} \leq y_{1} \text { and } x_{2} \leq y_{2}\right) \text {, for all }\left[x_{1}, x_{2}\right],\left[y_{1}, y_{2}\right] \text { in } L^{I} .}
\end{aligned}
$$

Similarly as Lemma 2.1 in [9] it can be shown that $\mathcal{L}^{I}$ is a complete lattice.

Definition $2[15,19]$ An interval-valued fuzzy set on $U$ is a mapping $A: U \rightarrow$ $L^{I}$.

Definition 3 [1] An intuitionistic fuzzy set on $U$ in Atanassov's sense is a set

$$
A=\left\{\left(u, \mu_{A}(u), \nu_{A}(u)\right) \mid u \in U\right\},
$$

where $\mu_{A}(u) \in[0,1]$ denotes the membership degree and $\nu_{A}(u) \in[0,1]$ the non-membership degree of $u$ in $A$ and where for all $u \in U, \mu_{A}(u)+\nu_{A}(u) \leq 1$.

An intuitionistic fuzzy set $A$ on $U$ in Atanassov's sense can be represented by the $\mathcal{L}^{I}$-fuzzy set $A$ given by

$$
\begin{aligned}
A: U & \rightarrow L^{I}: \\
u & \mapsto\left[\mu_{A}(u), 1-\nu_{A}(u)\right], \quad \forall u \in U .
\end{aligned}
$$

In Figure 1 the set $L^{I}$ is shown. Note that $x=\left[x_{1}, x_{2}\right] \in L^{I}$ is identified with the point $\left(x_{1}, x_{2}\right) \in \mathbb{R}^{2}$.

In the sequel, if $x \in L^{I}$, then we denote its bounds by $x_{1}$ and $x_{2}$, i.e. $x=$ $\left[x_{1}, x_{2}\right]$. The length $x_{2}-x_{1}$ of the interval $x \in L^{I}$ is called the degree of 


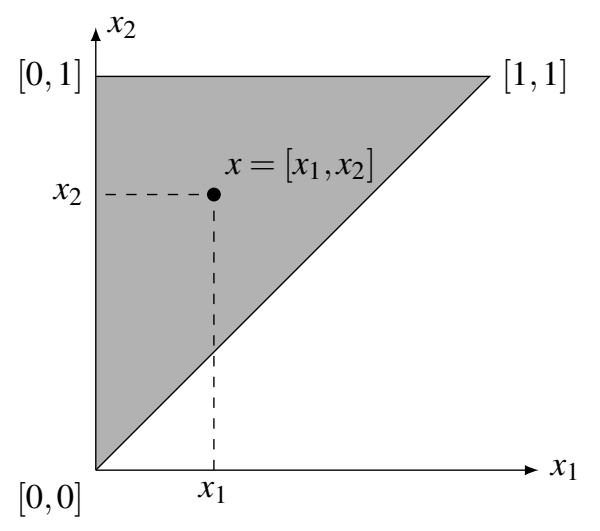

Figure 1. The grey area is $L^{I}$.

uncertainty and is denoted by $x_{\pi}$. The smallest and the largest element of $\mathcal{L}^{I}$ are given by $0_{\mathcal{L}^{I}}=[0,0]$ and $1_{\mathcal{L}^{I}}=[1,1]$. Note that, for $x, y$ in $L^{I}, x<_{L^{I}} y$ is equivalent to $x \leq_{L^{I}} y$ and $x \neq y$, i.e. either $x_{1}<y_{1}$ and $x_{2} \leq y_{2}$, or $x_{1} \leq y_{1}$ and $x_{2}<y_{2}$. We define the relation $\ll_{L^{I}}$ by $x \ll_{L^{I}} y \Longleftrightarrow x_{1}<y_{1}$ and $x_{2}<y_{2}$, for $x, y$ in $L^{I}$. The order $\leq_{L^{I}}$ is called in [12] the "weak truth ordering": interpreting an interval as a range between a pessimistic and an optimistic truth-evaluation of a proposition, this ordering tells us that one interval evaluation is considered smaller or equal than another one if it is the case for both the corresponding pessimistic and optimistic truth evaluations. This is not always the most accurate order: if two intervals overlap, then the real truth value approximated by the lower interval is not necessarily smaller than the real value approximated by the highest interval. Therefore in [12] a "strong truth ordering" is considered defined by $x \preceq_{L^{I}} y \Longleftrightarrow\left(x_{2} \leq y_{1}\right.$ or $x=y$ ). This order suffers however from the same problem: denote the truth value approximated by an interval $x$ by $\hat{x}$ and the truth value approximated by $y$ by $\hat{y}$, then $x=y$ does not necessarily mean that $x$ and $y$ approximate the same truth value and therefore $\hat{x}$ can actually be larger than $\hat{y}$. On the other hand, if $x=y$ then the probability that $\hat{x} \leq \hat{y}$ is the same as the probability that $\hat{x} \geq \hat{y}$. Using this interpretation the weak truth ordering is more natural than the strong truth ordering, because $x \leq_{L^{I}} y$ iff the probability that $\hat{x} \leq \hat{y}$ is larger than the probability that $\hat{x} \geq \hat{y}$. In [12] a third ordering is considered, the "imprecision order", which is induced by the set inclusion $(x \subseteq y \Longleftrightarrow$ $x_{1} \geq y_{1}$ and $\left.x_{2} \leq y_{2}\right)$ and which orders intervals according to how precise they are. 
We define for further usage the sets

$$
\begin{aligned}
D & =\left\{\left[x_{1}, x_{1}\right] \mid x_{1} \in[0,1]\right\}, \\
\bar{L}^{I} & =\left\{\left[x_{1}, x_{2}\right] \mid\left(x_{1}, x_{2}\right) \in \mathbb{R}^{2} \text { and } x_{1} \leq x_{2}\right\}, \\
\bar{D} & =\left\{\left[x_{1}, x_{1}\right] \mid x_{1} \in \mathbb{R}\right\} \\
\bar{L}_{+}^{I} & =\left\{\left[x_{1}, x_{2}\right] \mid\left(x_{1}, x_{2}\right) \in\left[0,+\infty\left[^{2} \text { and } x_{1} \leq x_{2}\right\},\right.\right. \\
\bar{D}_{+} & =\left\{\left[x_{1}, x_{1}\right] \mid x_{1} \in[0,+\infty[\},\right. \\
\bar{L}_{+, 0}^{I} & =\left\{\left[x_{1}, x_{2}\right] \mid\left(x_{1}, x_{2}\right) \in\right] 0,+\infty\left[^{2} \text { and } x_{1} \leq x_{2}\right\}, \\
\bar{D}_{+, 0} & =\left\{\left[x_{1}, x_{1}\right] \mid x_{1} \in\right] 0,+\infty[\} .
\end{aligned}
$$

Note that for any non-empty subset $A$ of $L^{I}$ it holds that

$$
\begin{aligned}
\sup A & =\left[\sup \left\{x_{1} \mid\left[x_{1}, x_{2}\right] \in A\right\}, \sup \left\{x_{2} \mid\left[x_{1}, x_{2}\right] \in A\right\}\right], \\
\inf A & =\left[\inf \left\{x_{1} \mid\left[x_{1}, x_{2}\right] \in A\right\}, \inf \left\{x_{2} \mid\left[x_{1}, x_{2}\right] \in A\right\}\right] .
\end{aligned}
$$

Definition $4 A$ t-norm on $\mathcal{L}^{I}$ is a commutative, associative, increasing mapping $\mathcal{T}:\left(L^{I}\right)^{2} \rightarrow L^{I}$ which satisfies $\mathcal{T}\left(1_{\mathcal{L}^{I}}, x\right)=x$, for all $x \in L^{I}$.

A t-conorm on $\mathcal{L}^{I}$ is a commutative, associative, increasing mapping $\mathcal{S}$ : $\left(L^{I}\right)^{2} \rightarrow L^{I}$ which satisfies $\mathcal{S}\left(0_{\mathcal{L}^{I}}, x\right)=x$, for all $x \in L^{I}$.

Definition $5 A$ t-norm $\mathcal{T}$ on $\mathcal{L}^{I}$ is called $t$-representable if there exist $t$-norms $T_{1}$ and $T_{2}$ on $([0,1], \leq)$ such that $T_{1}\left(x_{1}, y_{1}\right) \leq T_{2}\left(x_{1}, y_{1}\right)$, for all $x_{1}, y_{1}$ in $[0,1]$, and such that, for all $x, y$ in $L^{I}$,

$$
\mathcal{T}(x, y)=\left[T_{1}\left(x_{1}, y_{1}\right), T_{2}\left(x_{2}, y_{2}\right)\right] .
$$

Then $T_{1}$ and $T_{2}$ are called the representants of $\mathcal{T}$, and $\mathcal{T}$ is denoted by $\mathcal{T}_{T_{1}, T_{2}}$.

A t-norm $\mathcal{T}$ on $\mathcal{L}^{I}$ is called pseudo-t-representable if there exists a $t$-norm $T$ on $([0,1], \leq)$ such that, for all $x, y$ in $L^{I}$,

$$
\mathcal{T}(x, y)=\left[T\left(x_{1}, y_{1}\right), \max \left(T\left(x_{1}, y_{2}\right), T\left(x_{2}, y_{1}\right)\right)\right] .
$$

Then $T$ is called the representant of $\mathcal{T}$, and $\mathcal{T}$ is denoted by $\mathcal{T}_{T}$.

In $[8,10]$ the following classes of t-norms on $\mathcal{L}^{I}$ are introduced: let $T$ be a t-norm on $([0,1], \leq)$, then the mappings $\mathcal{T}_{T, t}$ and $\mathcal{T}_{T}^{\prime}$ given by, for all $x, y$ in $L^{I}$,

$$
\begin{aligned}
\mathcal{T}_{T, t}(x, y) & =\left[T\left(x_{1}, y_{1}\right), \max \left(T\left(t, T\left(x_{2}, y_{2}\right)\right), T\left(x_{1}, y_{2}\right), T\left(x_{2}, y_{1}\right)\right)\right] \\
\mathcal{T}_{T}^{\prime}(x, y) & =\left[\min \left(T\left(x_{1}, y_{2}\right), T\left(x_{2}, y_{1}\right)\right), T\left(x_{2}, y_{2}\right)\right]
\end{aligned}
$$

are t-norms on $\mathcal{L}^{I}$. This class $\mathcal{T}_{T, t}$ of t-norms is important in interval-valued fuzzy logic, since any t-norm on $\mathcal{L}^{I}$ which satisfies the residuation principle 
and for which the t-norm itself and its residual implication are both natural extensions (see below for the definition of natural extension) of corresponding operations on the unit interval, is an element of this class (see Theorem 15 in $[22])$.

Definition 6 A negation on $\mathcal{L}^{I}$ is a decreasing mapping $\mathcal{N}: L^{I} \rightarrow L^{I}$ for which $\mathcal{N}\left(0_{\mathcal{L}^{I}}\right)=1_{\mathcal{L}^{I}}$ and $\mathcal{N}\left(1_{\mathcal{L}^{I}}\right)=0_{\mathcal{L}^{I}}$. If $\mathcal{N}(\mathcal{N}(x))=x$, for all $x \in L^{I}$, then $\mathcal{N}$ is called involutive.

The mapping $\mathcal{N}_{s}$ defined by $\mathcal{N}_{s}(x)=\left[1-x_{2}, 1-x_{1}\right]$, for all $x \in L^{I}$, is a negation on $\mathcal{L}^{I}$ and is called the standard negation on $\mathcal{L}^{I}$. Note that $\mathcal{N}_{s}(x)=$ $\left[N_{s}\left(x_{2}\right), N_{s}\left(x_{1}\right)\right]$, where $N_{s}$ is the standard negation on $([0,1], \leq)$ given by $N_{s}\left(x_{1}\right)=1-x_{1}$, for all $x_{1} \in[0,1]$.

Let $\mathcal{T}$ be a t-norm and $\mathcal{N}$ an involutive negation on $\mathcal{L}^{I}$. Then the mapping $\mathcal{T}_{\mathcal{N}}^{*}:\left(L^{I}\right)^{2} \rightarrow L^{I}$ defined by, for all $x, y$ in $L^{I}, \mathcal{T}_{\mathcal{N}}^{*}(x, y)=\mathcal{N}(\mathcal{T}(\mathcal{N}(x), \mathcal{N}(y)))$, is a t-conorm on $\mathcal{L}^{I}$, called the dual t-conorm of $\mathcal{T}$ w.r.t. $\mathcal{N}$. Similarly the dual t-norm of a t-conorm w.r.t. an involutive negation on $\mathcal{L}^{I}$ is defined.

Let $n \in \mathbb{N} \backslash\{0\}$. If for an $n$-ary mapping $f$ on $[0,1]$ and an $n$-ary mapping $F$ on $L^{I}$ it holds that $F\left(\left[a_{1}, a_{1}\right], \ldots,\left[a_{n}, a_{n}\right]\right)=\left[f\left(a_{1}, \ldots, a_{n}\right), f\left(a_{1}, \ldots, a_{n}\right)\right]$, for all $\left(a_{1}, \ldots, a_{n}\right) \in[0,1]^{n}$, then we say that $F$ is a natural extension of $f$ to $L^{I}$. Clearly, for any mapping $F$ on $L^{I}, F(D, \ldots, D) \subseteq D$ if and only if there exists a mapping $f$ on $[0,1]$ such that $F$ is a natural extension of $f$ to $L^{I}$. E.g. $\mathcal{T}_{T, T}$, $\mathcal{T}_{T}, \mathcal{T}_{T, t}$ and $\mathcal{T}_{T}^{\prime}$ are all natural extensions of $T$ to $L^{I}, \mathcal{N}_{s}$ is a natural extension of $N_{s}$.

Example 7 Let, for all $x_{1}, y_{1}$ in $[0,1]$,

$$
\begin{aligned}
T_{W}\left(x_{1}, y_{1}\right) & =\max \left(0, x_{1}+y_{1}-1\right), \\
T_{P}\left(x_{1}, y_{1}\right) & =x_{1} y_{1}, \\
T_{D}\left(x_{1}, y_{1}\right) & = \begin{cases}\min \left(x_{1}, y_{1}\right), & \text { if } \max \left(x_{1}, y_{1}\right)=1, \\
0, & \text { else },\end{cases} \\
S_{W}\left(x_{1}, y_{1}\right) & =\min \left(1, x_{1}+y_{1}\right) .
\end{aligned}
$$

Then $T_{W}, T_{P}$ and $T_{D}$ are t-norms, and $S_{W}$ is a t-conorm on $([0,1], \leq)$. Let now, for all $x, y$ in $L^{I}$,

$$
\begin{aligned}
\mathcal{T}_{W}(x, y) & =\left[\max \left(0, x_{1}+y_{1}-1\right), \max \left(0, x_{1}+y_{2}-1, x_{2}+y_{1}-1\right)\right], \\
\mathcal{T}_{P}(x, y) & =\left[x_{1} y_{1}, \max \left(x_{1} y_{2}, x_{2} y_{1}\right)\right], \\
\mathcal{S}_{W}(x, y) & =\left[\min \left(1, x_{1}+y_{2}, x_{2}+y_{1}\right), x_{2}+y_{2}\right] .
\end{aligned}
$$

Then $\mathcal{T}_{W}$ and $\mathcal{T}_{P}$ are t-norms, and $\mathcal{S}_{W}$ is a t-conorm on $\mathcal{L}^{I}$. Furthermore, $\mathcal{T}_{W}$, $\mathcal{T}_{P}$ and $\mathcal{S}_{W}$ are natural extensions of $T_{W}, T_{P}$ and $S_{W}$ respectively. In $[2,4,7]$ it is shown that $\mathcal{T}_{W}$ inherits more interesting properties from the Eukasiewicz 
t-norm on the unit interval than the t-representable extension of $T_{W}$ to $L^{I}$.

\section{Arithmetic operators on $\bar{L}^{I}$}

We start from two arithmetic operators $\oplus:\left(\bar{L}^{I}\right)^{2} \rightarrow \bar{L}^{I}$ and $\otimes:\left(\bar{L}_{+}^{I}\right)^{2} \rightarrow \bar{L}^{I}$ satisfying the following properties,

$(\mathrm{ADD}-1) \oplus$ is commutative,

$($ ADD-2) $\oplus$ is associative,

(ADD-3) $\oplus$ is increasing,

$(\mathrm{ADD}-4) 0_{\mathcal{L}^{I}} \oplus a=a$, for all $a \in \bar{L}^{I}$,

$(\mathrm{ADD}-5)[\alpha, \alpha] \oplus[\beta, \beta]=[\alpha+\beta, \alpha+\beta]$, for all $\alpha, \beta$ in $[0,+\infty[$,

$($ MUL- 1$) \otimes$ is commutative,

$($ MUL-2) $\otimes$ is associative,

(MUL-3) $\otimes$ is increasing,

(MUL-4) $1_{\mathcal{L}^{I}} \otimes a=a$, for all $a \in \bar{L}_{+}^{I}$,

(MUL-5) $[\alpha, \alpha] \otimes[\beta, \beta]=[\alpha \beta, \alpha \beta]$, for all $\alpha, \beta$ in $[0,+\infty[$.

The conditions (ADD-1)-(ADD-4) and (MUL-1)-(MUL-4) are natural conditions for any addition and multiplication operators. The conditions (ADD-5) and (MUL-5) ensure that these operators are natural extensions of the addition and multiplication of real numbers to $\bar{L}^{I}$.

Sometimes we will assume that $\oplus$ and $\otimes$ satisfy the following conditions instead of (ADD-5) and (MUL-5):

(ADD-5') $[\alpha, \alpha] \oplus b=\left[\alpha+b_{1}, \alpha+b_{2}\right]$, for all $\alpha \in \mathbb{R}$ and $b \in \bar{L}^{I}$, (MUL-5') $[\alpha, \alpha] \otimes b=\left[\alpha b_{1}, \alpha b_{2}\right]$, for all $\alpha \in\left[0,+\infty\left[\right.\right.$ and $b \in \bar{L}_{+}^{I}$.

Note that from (ADD-3) and (ADD-4) it follows that, for all $a, b$ in $\bar{L}^{I}, a \oplus b \geq_{L^{I}}$ $a$, if $b \geq_{L^{I}} 0_{\mathcal{L}^{I}}$. Similarly, we find that $a \otimes b \geq_{L^{I}} a$, if $b \geq_{L^{I}} 1_{\mathcal{L}^{I}}$, for all $a, b$ in $\bar{L}_{+}^{I}$. On the other hand, in order to show that $\oplus$ satisfies (ADD-5') it is sufficient to prove that $[\alpha, \alpha] \oplus b=\left[\alpha+b_{1}, \alpha+b_{2}\right]$, for all $\left.\alpha \in\right] 0,+\infty[$ and $b \in \bar{L}^{I}$. Indeed, for $\left.\alpha \in\right]-\infty, 0\left[\right.$ and $b \in \bar{L}^{I}$, we obtain using (ADD-2), (ADD-4), (ADD-5) and (ADD-5') that

$$
\begin{aligned}
{\left[\alpha+b_{1}, \alpha+b_{2}\right] } & =0_{\mathcal{L}^{I} \oplus\left[\alpha+b_{1}, \alpha+b_{2}\right]} \\
& =[\alpha, \alpha] \oplus[-\alpha,-\alpha] \oplus\left[\alpha+b_{1}, \alpha+b_{2}\right] \\
& =[\alpha, \alpha] \oplus\left[-\alpha+\left(\alpha+b_{1}\right),-\alpha+\left(\alpha+b_{2}\right)\right] \\
& =[\alpha, \alpha] \oplus b .
\end{aligned}
$$

For $\alpha=0$ and $b \in \bar{L}^{I}$, it follows from (ADD-4) that $[0,0] \oplus b=\left[0+b_{1}, 0+b_{2}\right]$. Using a similar reasoning and using the fact that $0=[0,0] \otimes\left[b_{1}, b_{1}\right] \leq_{L^{I}}[0,0] \otimes$ 
$b \leq_{L^{I}}[0,0] \otimes\left[b_{2}, b_{2}\right]=0$, we obtain that (MUL-5') follows from $[\alpha, \alpha] \otimes b=$ $\left[\alpha b_{1}, \alpha b_{2}\right]$ for all $\left.\alpha \in\right] 1,+\infty\left[\right.$ and $b \in \bar{L}_{+}^{I}$.

Similarly as in (ADD-5') we want to define $1_{\mathcal{L}^{I}} \ominus x$ componentwise. In order to obtain a similar arithmetic formula for the standard negation on $\mathcal{L}^{I}$ as for the standard negation on the unit interval, we define for all $x \in \bar{L}^{I}$,

$$
1_{\mathcal{L}^{I}} \ominus x=\left[1-x_{2}, 1-x_{1}\right] .
$$

For general $x$ and $y$ in $\bar{L}^{I}$ we extend the subtraction using the connection between $\oplus_{\mathcal{L}^{I}}$ and $\ominus_{\mathcal{L}^{I}}$ (see Example 8) that was obtained in [6]: for all $x, y$ in $\bar{L}^{I}$,

$$
x \ominus y=1_{\mathcal{L}^{I}} \ominus\left(\left(1_{\mathcal{L}^{I}} \ominus x\right) \oplus y\right) .
$$

Similarly, we define the mapping $\oslash$ by, for all $x, y$ in $\bar{L}_{+, 0}^{I}$,

$$
1_{\mathcal{L}^{I}} \oslash x=\left[\frac{1}{x_{2}}, \frac{1}{x_{1}}\right]
$$

and

$$
x \oslash y=1_{\mathcal{L}^{I}} \oslash\left(\left(1_{\mathcal{L}^{I}} \oslash x\right) \otimes y\right) .
$$

Although there seems to be a duality between the addition and multiplication, this duality is not perfect, since we also allow the multiplication of elements for which the lower bound is 0 (as a consequence, for the division operator we have to exclude exactly those elements; such an exclusion does not occur for the subtraction). We allow these elements for the multiplication, because in this paper we want to extend the product t-norm to $\mathcal{L}^{I}$ using the multiplication.

Example 8 We give some examples of arithmetic operators satisfying the conditions (ADD-1)-(ADD-5) and (MUL-1)-(MUL-5).

- In the interval calculus (see e.g. [18]) the following operators are defined: for all $x, y$ in $\bar{L}^{I}$,

$$
\begin{aligned}
& x \oplus y=\left[x_{1}+y_{1}, x_{2}+y_{2}\right], \\
& x \ominus y=\left[x_{1}-y_{2}, x_{2}-y_{1}\right], \\
& x \otimes y=\left[x_{1} y_{1}, x_{2} y_{2}\right], \quad \text { if } x, y \text { in } \bar{L}_{+}^{I}, \\
& x \oslash y=\left[\frac{x_{1}}{y_{2}}, \frac{x_{2}}{y_{1}}\right], \quad \text { if } x, y \text { in } \bar{L}_{+, 0}^{I} .
\end{aligned}
$$

It is easy to see that these operators satisfy (ADD-1)-(ADD-5), (MUL-1)(MUL-5), (ADD-5'), (MUL-5'), (1), (2), (3) and (4). 
- In [6] the following operators are defined: for all $x, y$ in $\bar{L}^{I}$,

$$
\begin{aligned}
& x \bigoplus_{\mathcal{L}^{I}} y=\left[\min \left(x_{1}+y_{2}, x_{2}+y_{1}\right), x_{2}+y_{2}\right], \\
& x \ominus_{\mathcal{L}^{I}} y=\left[x_{1}-y_{2}, \max \left(x_{1}-y_{1}, x_{2}-y_{2}\right)\right], \\
& x \otimes_{\mathcal{L}^{I}} y=\left[x_{1} y_{1}, \max \left(x_{1} y_{2}, x_{2} y_{1}\right)\right], \quad \text { if } x, y \text { in } \bar{L}_{+}^{I}, \\
& x \oslash_{\mathcal{L}^{I}} y=\left[\min \left(\frac{x_{1}}{y_{1}}, \frac{x_{2}}{y_{2}}\right), \frac{x_{2}}{y_{1}}\right], \quad \text { if } x, y \text { in } \bar{L}_{+, 0}^{I} .
\end{aligned}
$$

It was proven in [6] that these operators satisfy (ADD-1)-(ADD-5), (MUL-1)(MUL-5), (ADD-5'), (MUL-5'), (1), (2), (3) and (4). In [5] these operators are used to define additive and multiplicative generators on $\mathcal{L}^{I}$ and it is shown that the only t-norms that can have a continuous additive generator based on this addition are t-representable t-norms.

- In [11] the following operators are defined for all $t \in] 0,1]$ : for all $x, y$ in $\bar{L}^{I}$,

$$
\begin{aligned}
& x \oplus_{\mathcal{L}^{I}}^{t} y=\left[\min \left(1-t+x_{1}+y_{1}, x_{1}+y_{2}, x_{2}+y_{1}\right), x_{2}+y_{2}\right], \\
& x \ominus_{\mathcal{L}^{I}}^{t} y=\left[x_{1}-y_{2}, \max \left(t+x_{2}-y_{1}-1, x_{1}-y_{1}, x_{2}-y_{2}\right)\right], \\
& x \otimes_{\mathcal{L}^{I}}^{t} y=\left[x_{1} y_{1}, \max \left(t x_{2} y_{2}, x_{1} y_{2}, x_{2} y_{1}\right)\right], \quad \text { if } x, y \text { in } \bar{L}_{+}^{I}, \\
& x \oslash_{\mathcal{L}^{I}}^{t} y=\left[\min \left(\frac{x_{1}}{t y_{2}}, \frac{x_{1}}{y_{1}}, \frac{x_{2}}{y_{2}}\right), \frac{x_{2}}{y_{1}}\right], \quad \text { if } x, y \text { in } \bar{L}_{+, 0}^{I} .
\end{aligned}
$$

It was proven in [11] that these operators satisfy (ADD-1)-(ADD-5), (MUL$1)-($ MUL-5), (ADD-5’), (MUL-5'), (1) and (2). We prove (2) and (4). For any $x, y$ in $\bar{L}^{I}$ we obtain

$$
\begin{aligned}
& 1_{\mathcal{L}^{I}} \ominus_{\mathcal{L}^{I}}^{t}\left(\left(1_{\mathcal{L}^{I}} \ominus_{\mathcal{L}^{I}}^{t} x\right) \oplus_{\mathcal{L}^{I}}^{t} y\right) \\
& =1_{\mathcal{L}^{I}} \ominus_{\mathcal{L}^{I}}^{t}\left[\min \left(1-t+1-x_{2}+y_{1}, 1-x_{2}+y_{2}, 1-x_{1}+y_{1}\right), 1-x_{1}+y_{2}\right] \\
& =\left[x_{1}-y_{2}, \max \left(t+x_{2}-y_{1}-1, x_{2}-y_{2}, x_{1}-y_{1}\right)\right] \\
& =x \ominus_{\mathcal{L}^{I}}^{t} y
\end{aligned}
$$

and similarly for (4).

- Define the following operators, for all $x, y$ in $\bar{L}^{I}$,

$$
\begin{aligned}
& x \oplus_{\mathcal{L}^{I}}^{\prime} y=\left[x_{1}+y_{1}, \max \left(x_{1}+y_{2}, x_{2}+y_{1}\right)\right], \\
& x \ominus_{\mathcal{L}^{I}}^{\prime} y=\left[\min \left(x_{1}-y_{1}, x_{2}-y_{2}\right), x_{2}-y_{1}\right], \\
& x \otimes_{\mathcal{L}^{I}}^{\prime} y=\left[\min \left(x_{1} y_{2}, x_{2} y_{1}\right), x_{2} y_{2}\right], \quad \text { if } x, y \text { in } \bar{L}_{+}^{I}, \\
& x \oslash_{\mathcal{L}^{I}}^{\prime} y=\left[\frac{x_{1}}{y_{2}}, \max \left(\frac{x_{1}}{y_{1}}, \frac{x_{2}}{y_{2}}\right)\right], \quad \text { if } x, y \text { in } \bar{L}_{+, 0}^{I} .
\end{aligned}
$$

It is easy to verify that these operators satisfy (ADD-1)-(ADD-5), (MUL-1)(MUL-5), (ADD-5'), (MUL-5'), (1), (2), (3) and (4). 
- Define the following operators, for all $x, y$ in $\bar{L}^{I}$,

$x \tilde{\oplus}_{\mathcal{L}^{I}} y=\left[\min \left(2 x_{2}+y_{1}, 2 y_{2}+x_{1}, x_{2}+y_{2}\right), x_{2}+y_{2}\right]$,

$x \tilde{\ominus}_{\mathcal{L}^{I}} y=\left[x_{1}-y_{2}, \max \left(2 x_{1}-y_{1}-1, x_{2}-2 y_{2}, x_{1}-y_{2}\right)\right]$,

$x \tilde{\otimes}_{\mathcal{L}^{I}} y=\left[x_{1} y_{1}, \max \left(\left(x_{2}+1\right) y_{1}-1,\left(y_{2}+1\right) x_{1}-1, x_{1} y_{1}\right)\right], \quad$ if $x, y$ in $\bar{L}_{+}^{I}$,

$x \tilde{\oslash}_{\mathcal{L}^{I}} y=\left[\min \left(\frac{x_{1}}{\left(x_{1}+1\right) y_{1}-x_{1}}, \frac{x_{2}}{y_{2}+1-x_{2}}, \frac{x_{2}}{y_{1}}\right), \frac{x_{2}}{y_{1}}\right], \quad$ if $x, y$ in $\bar{L}_{+, 0}^{I}$.

It can be verified that these operators satisfy (ADD-1)-(ADD-5), (MUL-1)(MUL-5), (1), (2), (3) and (4). For example we prove (2): for any $x, y$ in $\bar{L}^{I}$ we obtain

$$
\begin{aligned}
& 1_{\mathcal{L}^{I}} \tilde{\theta}_{\mathcal{L}^{I}}\left(\left(1_{\mathcal{L}^{I}} \tilde{\theta}_{\mathcal{L}^{I}} x\right) \tilde{\oplus}_{\mathcal{L}^{I}} y\right) \\
& =1_{\mathcal{L}^{I}} \tilde{\theta}_{\mathcal{L}^{I}}\left(\left[1-x_{2}, 1-x_{1}\right] \tilde{\oplus}_{\mathcal{L}^{I}} y\right) \\
& =1_{\mathcal{L}^{I}} \tilde{\theta}_{\mathcal{L}^{I}}\left[\min \left(2-2 x_{1}+y_{1}, 2 y_{2}+1-x_{2}, 1-x_{1}+y_{2}\right), 1-x_{1}+y_{2}\right] \\
& =\left[x_{1}-y_{2}, \max \left(2 x_{1}-y_{1}-1, x_{2}-2 y_{2}, x_{1}-y_{2}\right)\right] \\
& =x \tilde{\theta}_{\mathcal{L}^{I}} y .
\end{aligned}
$$

On the other hand, $\tilde{\oplus}_{\mathcal{L}^{I}}$ does not satisfy (ADD-5') and $\tilde{\otimes}_{\mathcal{L}^{I}}$ does not satisfy (MUL-5'): e.g. for $\tilde{\oplus}_{\mathcal{L}^{I}}$ we obtain that $[1,1] \tilde{\oplus}_{\mathcal{L}^{I}} x=\left[\min \left(2+x_{1}, 1+x_{2}\right), 1+x_{2}\right]$ is in general not equal to $\left[1+x_{1}, 1+x_{2}\right]$.

Theorem 9 The mapping $\ominus$ satisfies the following properties, for all $\alpha, \beta$ in $\mathbb{R}$ and $a, b, c$ in $\bar{L}^{I}$,

(i) $\ominus$ is increasing in its first and decreasing in its second argument,

(ii) $[\alpha, \alpha] \ominus[\beta, \beta]=[\alpha-\beta, \alpha-\beta]$,

(iii) $a \oplus b=1_{\mathcal{L}^{I}} \ominus\left(\left(1_{\mathcal{L}^{I}} \ominus a\right) \ominus b\right)$,

(iv) $(a \ominus b) \ominus c=a \ominus(b \oplus c)=(a \ominus c) \ominus b$,

(v) $a \ominus b=\left(1_{\mathcal{L}^{I}} \ominus b\right) \ominus\left(1_{\mathcal{L}^{I}} \ominus a\right)$,

(vi) $a \ominus\left(1_{\mathcal{L}^{I}} \ominus b\right)=b \ominus\left(1_{\mathcal{L}^{I}} \ominus a\right)$,

(vii) $a \ominus(b \ominus c)=\left(\left(1_{\mathcal{L}^{I}} \ominus b\right) \oplus c\right) \ominus\left(1_{\mathcal{L}^{I}} \ominus a\right)$.

If $\oplus$ satisfies (ADD-5'), then

$$
\begin{aligned}
& \text { (viii) }[\alpha, \alpha] \ominus b=\left[\alpha-b_{2}, \alpha-b_{1}\right], \\
& \text { (ix) } b \ominus[\alpha, \alpha]=\left[b_{1}-\alpha, b_{2}-\alpha\right], \\
& \text { (x) } a \ominus b=[\alpha, \alpha] \ominus(([\alpha, \alpha] \ominus a) \oplus b), \\
& \text { (xi) } a \oplus b=[\alpha, \alpha] \ominus(([\alpha, \alpha] \ominus a) \ominus b), \\
& \text { (xii) }(a \oplus b) \ominus[\alpha, \alpha]=a \oplus(b \ominus[\alpha, \alpha])=b \oplus(a \ominus[\alpha, \alpha]) \text {. }
\end{aligned}
$$

The mapping $\oslash$ satisfies the following properties, for all $\alpha, \beta$ in $] 0,+\infty[$ and $a, b, c$ in $\bar{L}_{+, 0}^{I}$,

(xiii) $\oslash$ is increasing in its first and decreasing in its second argument, 


$$
\begin{aligned}
& \text { (xiv) }[\alpha, \alpha] \oslash[\beta, \beta]=\left[\frac{\alpha}{\beta}, \frac{\alpha}{\beta}\right] \text {, } \\
& \text { (xv) }(a \oslash b) \oslash c=a \oslash(b \otimes c)=(a \oslash c) \oslash b \text {, } \\
& \text { (xvi) } a \otimes b=1_{\mathcal{L}^{I}} \oslash\left(\left(1_{\mathcal{L}^{I}} \oslash a\right) \oslash b\right) \text {, } \\
& \text { (xvii) } a \oslash b=\left(1_{\mathcal{L}^{I}} \oslash b\right) \oslash\left(1_{\mathcal{L}^{I}} \oslash a\right) \text {, } \\
& \text { (xviii) } a \oslash\left(1_{\mathcal{L}^{I}} \oslash b\right)=b \oslash\left(1_{\mathcal{L}^{I}} \oslash a\right) \text {, } \\
& \text { (xix) } a \oslash(b \oslash c)=\left(\left(1_{\mathcal{L}^{I}} \oslash b\right) \otimes c\right) \oslash\left(1_{\mathcal{L}^{I}} \oslash a\right) \text {. }
\end{aligned}
$$

If $\otimes$ satisfies (MUL-5'), then

$$
\begin{aligned}
& \text { (xx) }[\alpha, \alpha] \oslash b=\left[\frac{\alpha}{b_{2}}, \frac{\alpha}{b_{1}}\right], \\
& \text { (xxi) } b \oslash[\alpha, \alpha]=\left[\frac{b_{1}}{\alpha}, \frac{b_{2}}{\alpha}\right], \\
& \text { (xxii) } a \oslash b=[\alpha, \alpha] \oslash(([\alpha, \alpha] \oslash a) \otimes b) . \\
& \text { (xxiii) } a \otimes b=[\alpha, \alpha] \oslash(([\alpha, \alpha] \oslash a) \oslash b), \\
& \text { (xxiv) }(a \otimes b) \oslash[\alpha, \alpha]=a \otimes(b \oslash[\alpha, \alpha])=b \otimes(a \oslash[\alpha, \alpha]) .
\end{aligned}
$$

PROOF. Let $\alpha, \beta$ in $\mathbb{R}$ and $a, b, c$ in $\bar{L}^{I}$.

(ii) Using the definition of $\ominus$ and (ADD-5), we obtain $[\alpha, \alpha] \ominus[\beta, \beta]=1_{\mathcal{L}^{I}} \ominus$ $([1-\alpha, 1-\alpha] \oplus[\beta, \beta])=1_{\mathcal{L}^{I}} \ominus[1-\alpha+\beta, 1-\alpha+\beta]=[\alpha-\beta, \alpha-\beta]$.

(iii) $1_{\mathcal{L}^{I}} \ominus\left(\left(1_{\mathcal{L}^{I}} \ominus a\right) \ominus b\right)=1_{\mathcal{L}^{I}} \ominus\left(1_{\mathcal{L}^{I}} \ominus\left(\left(1_{\mathcal{L}^{I}} \ominus\left(1_{\mathcal{L}^{I}} \ominus a\right)\right) \oplus b\right)\right)$

$$
=a \oplus b
$$

using the definition of $\ominus$ and the fact that $1_{\mathcal{L}^{I}} \ominus\left(1_{\mathcal{L}^{I}} \ominus x\right)=x$, for all $x \in \bar{L}^{I}$.

(iv) $(a \ominus b) \ominus c=1_{\mathcal{L}^{I}} \ominus\left(\left(1_{\mathcal{L}^{I}} \ominus(a \ominus b)\right) \oplus c\right)$

$$
\begin{aligned}
& =1_{\mathcal{L}^{I}}\left(\left(\left(1_{\mathcal{L}^{I}} \ominus a\right) \oplus b\right) \oplus c\right) \\
& =1_{\mathcal{L}^{I}}\left(\left(1_{\mathcal{L}^{I}} \ominus a\right) \oplus(b \oplus c)\right) \\
& =a \ominus(b \oplus c),
\end{aligned}
$$

using the associativity of $\oplus$ and the fact that $1_{\mathcal{L}^{I}} \ominus\left(1_{\mathcal{L}^{I}} \ominus x\right)=x$, for all $x \in \bar{L}^{I}$.

Since the expression $a \ominus(b \oplus c)$ is symmetrical in $b$ and $c$, it follows that $(a \ominus b) \ominus c=(a \ominus c) \ominus b$.

(v) $a \ominus b=1_{\mathcal{L}^{I}} \ominus\left(\left(1_{\mathcal{L}^{I}} \ominus a\right) \oplus b\right)$

$$
\begin{aligned}
& =1_{\mathcal{L}^{I}} \ominus\left(\left(1_{\mathcal{L}^{I}} \ominus\left(1_{\mathcal{L}^{I}} \ominus b\right)\right) \oplus\left(1_{\mathcal{L}^{I}} \ominus a\right)\right) \\
& =\left(1_{\mathcal{L}^{I}} \ominus b\right) \ominus\left(1_{\mathcal{L}^{I}} \ominus a\right),
\end{aligned}
$$

using the commutativity of $\oplus$ and the fact that $1_{\mathcal{L}^{I}} \ominus\left(1_{\mathcal{L}^{I}} \ominus x\right)=x$, for all $x \in \bar{L}^{I}$.

(vi) Using the definition of $\ominus$, we obtain $a \ominus\left(1_{\mathcal{L}^{I}} \ominus b\right)=1_{\mathcal{L}^{I}} \ominus\left(\left(1_{\mathcal{L}^{I}} \ominus a\right) \oplus\left(1_{\mathcal{L}^{I}} \ominus\right.\right.$ $b)$, which is symmetrical in $a$ and $b$. Hence $a \ominus\left(1_{\mathcal{L}^{I}} \ominus b\right)=b \ominus\left(1_{\mathcal{L}^{I}} \ominus a\right)$. 
(vii) $a \ominus(b \ominus c)=1_{\mathcal{L}^{I}} \ominus\left(\left(1_{\mathcal{L}^{I}} \ominus a\right) \oplus\left(1_{\mathcal{L}^{I}} \ominus\left(\left(1_{\mathcal{L}^{I}} \ominus b\right) \oplus c\right)\right)\right)$

$$
\begin{aligned}
& =1_{\mathcal{L}^{I}} \ominus\left(\left(1_{\mathcal{L}^{I}} \ominus\left(\left(1_{\mathcal{L}^{I}} \ominus b\right) \oplus c\right)\right) \oplus\left(1_{\mathcal{L}^{I}} \ominus a\right)\right) \\
& =\left(\left(1_{\mathcal{L}^{I}} \ominus b\right) \oplus c\right) \ominus\left(1_{\mathcal{L}^{I}} \ominus a\right) .
\end{aligned}
$$

(viii) $[\alpha, \alpha] \ominus b=1_{\mathcal{L}^{I}} \ominus([1-\alpha, 1-\alpha] \oplus b)$

$$
\begin{aligned}
& =1_{\mathcal{L}^{I}} \ominus\left[1-\alpha+b_{1}, 1-\alpha+b_{2}\right] \\
& =\left[\alpha-b_{2}, \alpha-b_{1}\right] .
\end{aligned}
$$

(x) From (viii) it follows that $[\alpha, \alpha] \ominus([\alpha, \alpha] \ominus x)=x$, for all $x \in \bar{L}^{I}$. Using (iv) we obtain:

$$
\begin{aligned}
a \ominus b & =([\alpha, \alpha] \ominus([\alpha, \alpha] \ominus a)) \ominus b \\
& =[\alpha, \alpha] \ominus(([\alpha, \alpha] \ominus a) \oplus b) .
\end{aligned}
$$

(xi) From (viii) it follows that $[\alpha, \alpha] \ominus([\alpha, \alpha] \ominus x)=x$, for all $x \in \bar{L}^{I}$. Using (x) we obtain:

$$
\begin{aligned}
& {[\alpha, \alpha] \ominus(([\alpha, \alpha] \ominus a) \ominus b)} \\
& =[\alpha, \alpha] \ominus([\alpha, \alpha] \ominus(([\alpha, \alpha] \ominus([\alpha, \alpha] \ominus a)) \oplus b)) \\
& =[\alpha, \alpha] \ominus([\alpha, \alpha] \ominus(a \oplus b)) \\
& =a \oplus b .
\end{aligned}
$$

(xii) Using (viii) and (ix), we obtain that $0_{\mathcal{L}^{I}} \ominus(b \ominus[\alpha, \alpha])=0_{\mathcal{L}^{I}} \ominus\left[b_{1}-\alpha, b_{2}-\right.$ $\alpha]=\left[\alpha-b_{2}, \alpha-b_{1}\right]=[\alpha, \alpha] \ominus b$. So,

$$
\begin{aligned}
& (a \oplus b) \ominus[\alpha, \alpha] \\
& =(b \oplus a) \ominus[\alpha, \alpha] \\
& =([\alpha, \alpha] \ominus(([\alpha, \alpha] \ominus b) \ominus a)) \ominus[\alpha, \alpha] \quad \text { (using (xi)) } \\
& =\left[\alpha-(([\alpha, \alpha] \ominus b) \ominus a)_{2}-\alpha, \alpha-(([\alpha, \alpha] \ominus b) \ominus a)_{1}-\alpha\right] \quad \text { (using (viii) and (ix)) } \\
& =0_{\mathcal{L}^{I}} \ominus(([\alpha, \alpha] \ominus b) \ominus a) \quad \text { (using (viii)) } \\
& =0_{\mathcal{L}^{I}} \ominus\left(\left(0_{\mathcal{L}^{I}} \ominus(b \ominus[\alpha, \alpha])\right) \ominus a\right) \quad \text { (see above) } \\
& =(b \ominus[\alpha, \alpha]) \oplus a \quad \text { (using (xi)) } \\
& =a \oplus(b \ominus[\alpha, \alpha]) \text {. }
\end{aligned}
$$

Using (ADD-1) we obtain that $a \oplus(b \ominus[\alpha, \alpha])=(a \oplus b) \ominus[\alpha, \alpha]=(b \oplus$ a) $\ominus[\alpha, \alpha]=b \oplus(a \ominus[\alpha, \alpha])$.

The other properties are shown in a similar way.

We check whether a binary operator $\oplus$ on $\bar{L}^{I}$ can be found such that $\left(\bar{L}^{I}, \oplus\right.$, 
$0_{\mathcal{L}^{I}}$ is a group. First we give some lemmas.

Lemma 10 Assume that a mapping $\oplus:\left(\bar{L}^{I}\right)^{2} \rightarrow \bar{L}^{I}$ satisfies (ADD-1)-(ADD5) and $\left(\bar{L}^{I}, \oplus, 0_{\mathcal{L}^{I}}\right)$ is a group. Then for each element $x \in \bar{L}^{I}$ the inverse $x^{-1}$ is given by $x^{-1}=\left[-x_{2},-x_{1}\right]$.

PROOF. Assume that $\left(\bar{L}^{I}, \oplus\right)$ is a group. Let arbitrarily $x \in \bar{L}^{I}$. Then there exists an element $x^{-1} \in \bar{L}^{I}$ such that $x \oplus x^{-1}=0_{\mathcal{L}^{I}}$. Since $\oplus$ satisfies (ADD$2)-($ ADD-5), we obtain successively that

$$
\begin{aligned}
& x \oplus\left[\left(x^{-1}\right)_{1},\left(x^{-1}\right)_{1}\right] \leq_{L^{I}} 0_{\mathcal{L}^{I}}=x \oplus x^{-1} \\
& x \oplus\left[\left(x^{-1}\right)_{1},\left(x^{-1}\right)_{1}\right] \oplus\left[-\left(x^{-1}\right)_{1},-\left(x^{-1}\right)_{1}\right] \leq_{L^{I}} 0_{\mathcal{L}^{I}} \oplus\left[-\left(x^{-1}\right)_{1},-\left(x^{-1}\right)_{1}\right] \\
& x \leq_{L^{I}}\left[-\left(x^{-1}\right)_{1},-\left(x^{-1}\right)_{1}\right] \\
& x_{2} \leq-\left(x^{-1}\right)_{1} .
\end{aligned}
$$

Noting that $x^{-1} \leq_{L^{I}}\left[\left(x^{-1}\right)_{2},\left(x^{-1}\right)_{2}\right]$, we obtain similarly that $-\left(x^{-1}\right)_{2} \leq x_{1}$, so $x_{2}+\left(x^{-1}\right)_{1} \leq 0 \leq x_{1}+\left(x^{-1}\right)_{2}$. Similarly, from $\left[x_{1}, x_{1}\right] \oplus x^{-1} \leq_{L^{I}} x \oplus$ $x^{-1} \leq_{L^{I}}\left[x_{2}, x_{2}\right] \oplus x^{-1}$ it follows that $x_{1}+\left(x^{-1}\right)_{2} \leq 0 \leq x_{2}+\left(x^{-1}\right)_{1}$. Hence $x_{1}+\left(x^{-1}\right)_{2}=x_{2}+\left(x^{-1}\right)_{1}=0$, so $x^{-1}=\left[-x_{2},-x_{1}\right]$.

Lemma 11 Assume that a mapping $\oplus:\left(\bar{L}^{I}\right)^{2} \rightarrow \bar{L}^{I}$ satisfies (ADD-1)-(ADD5) and $\left(\bar{L}^{I}, \oplus, 0_{\mathcal{L}^{I}}\right)$ is a group. Define $\bar{D}^{\prime}=\{[-x, x] \mid x \in[0,+\infty[\}$. Then, for all $x \in \bar{L}^{I}$,

$$
x \in \bar{D}^{\prime} \Longleftrightarrow x \oplus x=0_{\mathcal{L}^{I}} .
$$

PROOF. Let arbitrarily $x \in \bar{L}^{I}$. From Lemma 10 it follows that $x^{-1}=$ $\left[-x_{2},-x_{1}\right]$. If $x \in \bar{D}^{\prime}$, then $x=x^{-1}=\left[-x_{2}, x_{2}\right]$, so $x \oplus x=x \oplus x^{-1}=0_{\mathcal{L}^{I}}$.

Assume conversely that $x \oplus x=0_{\mathcal{L}^{I}}$, then $x^{-1} \oplus x \oplus x=x^{-1} \oplus 0_{\mathcal{L}^{I}}$, so $\left[x_{1}, x_{2}\right]=0_{\mathcal{L}^{I}} \oplus x=x^{-1}=\left[-x_{2},-x_{1}\right]$. Thus $x_{1}=-x_{2}$, and hence $x \in \overline{D^{\prime}}$.

Theorem 12 Assume that a mapping $\oplus:\left(\bar{L}^{I}\right)^{2} \rightarrow \bar{L}^{I}$ satisfies (ADD-1)(ADD-5). Then $\left(\bar{L}^{I}, \oplus, 0_{\mathcal{L}^{I}}\right)$ is not a group.

PROOF. Assume that $\left(\bar{L}^{I}, \oplus, 0_{\mathcal{L}^{I}}\right)$ is a group. Let arbitrarily $x$ in $\bar{D}^{\prime}=$ $\left\{\left[-x_{2}, x_{2}\right] \mid x_{2} \in\left[0,+\infty[\}\right.\right.$ such that $x_{2}>0$. Let $y=x \oplus\left[x_{2}, x_{2}\right]$, then since $\oplus$ satisfies (ADD-3)-(ADD-5), we have that $y \geq_{L^{I}} x \oplus 0_{\mathcal{L}^{I}}=x$ and $y \geq_{L^{I}}\left[-x_{2},-x_{2}\right] \oplus\left[x_{2}, x_{2}\right]=0_{\mathcal{L}^{I}}$, so $y \geq_{L^{I}} \sup \left(x, 0_{\mathcal{L}^{I}}\right)=\left[0, x_{2}\right]$.

Let $z=x \oplus\left[0, x_{2}\right]$. Then $z \leq_{L^{I}} x \oplus y=x \oplus\left(x \oplus\left[x_{2}, x_{2}\right]\right)=(x \oplus x) \oplus\left[x_{2}, x_{2}\right]=$ $\left[x_{2}, x_{2}\right]$ (using Lemma 11), so $z_{2} \leq x_{2}$. We prove now that $z_{2}=x_{2}$. Assume that $z_{2}<x_{2}$. We first show that $(x \oplus z)_{1}<0$. Assume that this is not the case, then $x \oplus z \geq_{L^{I}} 0_{\mathcal{L}^{I}}$, so $z=(x \oplus x) \oplus z=x \oplus(x \oplus z) \geq_{L^{I}} x \oplus 0_{\mathcal{L}^{I}}=x$ (using Lemma 
11). Thus we obtain that $z_{2} \geq x_{2}$, which is a contradiction. Hence $(x \oplus z)_{1}<0$. But from this it follows that $\left(\left[0, x_{2}\right]\right)_{1}=\left(x \oplus x \oplus\left[0, x_{2}\right]\right)_{1}=(x \oplus z)_{1}<0$, which is a contradiction. Therefore, the assumption that $z_{2}<x_{2}$ is incorrect.

We now prove that $z=\left[0, x_{2}\right]$. Assume that $z_{1}>0$. Then, using the fact that $z_{2}=x_{2}$, we obtain that $\left[0, x_{2}\right]=x \oplus x \oplus\left[0, x_{2}\right]=x \oplus z \geq_{L^{I}} x \oplus\left[0, x_{2}\right]=z$, so $z_{1} \leq 0$, which is a contradiction. Similarly, the assumption $z_{1}<0$ leads to a contradiction, so $z_{1}=0$. Hence $x \oplus\left[0, x_{2}\right]=\left[0, x_{2}\right]$.

Now we obtain that $x=x \oplus\left[0, x_{2}\right] \oplus\left[0, x_{2}\right]^{-1}=\left[0, x_{2}\right] \oplus\left[0, x_{2}\right]^{-1}=0_{\mathcal{L}^{I}}$, which contradicts our choice of $x$. So $\left(\bar{L}^{I}, \oplus, 0_{\mathcal{L}^{I}}\right)$ is not a group.

Similarly we obtain the following lemmas and theorem.

Lemma 13 Assume that a mapping $\otimes:\left(\bar{L}_{+}^{I}\right)^{2} \rightarrow \bar{L}_{+}^{I}$ satisfies (MUL-1)-(MUL$5)$ and $\left(\bar{L}_{+, 0}^{I}, \otimes, 1_{\mathcal{L}^{I}}\right)$ is a group. Then for each element $x \in \bar{L}_{+, 0}^{I}$ the inverse $x^{-1}$ is given by $x^{-1}=\left[\frac{1}{x_{2}}, \frac{1}{x_{1}}\right]$.

Lemma 14 Assume that a mapping $\otimes:\left(\bar{L}_{+}^{I}\right)^{2} \rightarrow \bar{L}_{+}^{I}$ satisfies (MUL-1)-(MUL5) and $\left(\bar{L}_{+, 0}^{I}, \otimes, 1_{\mathcal{L}^{I}}\right)$ is a group. Define $\bar{D}^{\prime \prime}=\left\{\left[\frac{1}{x}, x\right] \mid x \in[1,+\infty[\}\right.$. Then, for all $x \in \bar{L}_{+, 0}^{I}$,

$$
x \in \bar{D}^{\prime \prime} \Longleftrightarrow x \otimes x=1_{\mathcal{L}^{I}}
$$

Theorem 15 Assume that a mapping $\otimes:\left(\bar{L}_{+}^{I}\right)^{2} \rightarrow \bar{L}_{+}^{I}$ satisfies (MUL-1)(MUL-5). Then $\left(\bar{L}_{+, 0}^{I}, \otimes, 1_{\mathcal{L}^{I}}\right)$ is not a group.

\section{The arithmetic operators and t-norms and t-conorms on $\mathcal{L}^{I}$}

Using two arithmetic operators $\oplus$ and $\otimes$ which satisfy (ADD-1)-(ADD-5) and (MUL-1)-(MUL-5), some t-norms and t-conorms can be deduced. Since the Eukasiewicz t-norm and t-conorm and the product t-norm on the unit interval play an important role in fuzzy set theory (see e.g. $[16,17]$ ), we construct tnorms and t-conorms on $\mathcal{L}^{I}$ using a similar algebraical expression as for the corresponding operations on the unit interval. In an example we will show that using some specific arithmetic operators, we obtain some well-known t(co)norms on $\mathcal{L}^{I}$.

Theorem 16 The mapping $\mathcal{S}_{\oplus}:\left(L^{I}\right)^{2} \rightarrow L^{I}$ defined by, for all $x, y$ in $L^{I}$,

$$
\mathcal{S}_{\oplus}(x, y)=\inf \left(1_{\mathcal{L}^{I}}, x \oplus y\right)
$$


is a t-conorm on $\mathcal{L}^{I}$ if and only if $\oplus$ satisfies the following condition:

$$
\begin{aligned}
& \left(\forall(x, y, z) \in\left(L^{I}\right)^{3}\right) \\
& \left(\left(\left(\inf \left(1_{\mathcal{L}^{I}}, x \oplus y\right) \oplus z\right)_{1}<1 \text { and }(x \oplus y)_{2}>1\right)\right. \\
& \left.\Longrightarrow\left(\inf \left(1_{\mathcal{L}^{I}}, x \oplus y\right) \oplus z\right)_{1}=\left(x \oplus \inf \left(1_{\mathcal{L}^{I}}, y \oplus z\right)\right)_{1}\right) .
\end{aligned}
$$

Furthermore $\mathcal{S}_{\oplus}$ is a natural extension of $S_{W}$ to $L^{I}$.

PROOF. Define the mapping $\mathcal{S}_{\oplus}:\left(L^{I}\right)^{2} \rightarrow L^{I}$ by $\mathcal{S}_{\oplus}(x, y)=\inf \left(1_{\mathcal{L}^{I}}, x \oplus y\right)$, for all $x, y$ in $L^{I}$. Clearly if $\mathcal{S}_{\oplus}$ is a t-conorm on $\mathcal{L}^{I}$, then from the associativity of $\mathcal{S}_{\oplus}$ it follows that $\left(\inf \left(1_{\mathcal{L}^{I}}, x \oplus y\right) \oplus z\right)_{1}=\left(x \oplus \inf \left(1_{\mathcal{L}^{I}}, y \oplus z\right)\right)_{1}$, for all $x, y, z$ in $L^{I}$ such that $\left(\inf \left(1_{\mathcal{L}^{I}}, x \oplus y\right) \oplus z\right)_{1}<1$. Hence $(6)$ holds.

Assume conversely that (6) holds. Then we prove that $\mathcal{S}_{\oplus}$ is a t-conorm. It is easy to see that $\mathcal{S}_{\oplus}$ is commutative and increasing, and $\mathcal{S}_{\oplus}\left(0_{\mathcal{L}^{I}}, x\right)=x$, for all $x \in L^{I}$. Finally we check the associativity. Let arbitrarily $x, y, z$ in $L^{I}$. We have the following cases.

(1) $\inf \left(1_{\mathcal{L}^{I}}, y \oplus z\right)=y \oplus z$ : in this case $\mathcal{S}_{\oplus}\left(x, \mathcal{S}_{\oplus}(y, z)\right)=\inf \left(1_{\mathcal{L}^{I}}, x \oplus(y \oplus z)\right)$.

(a) If $\inf \left(1_{\mathcal{L}^{I}}, x \oplus y\right)=x \oplus y$, then $\mathcal{S}_{\oplus}\left(\mathcal{S}_{\oplus}(x, y), z\right)=\inf \left(1_{\mathcal{L}^{I}},(x \oplus y) \oplus z\right)=$ $\mathcal{S}_{\oplus}\left(x, \mathcal{S}_{\oplus}(y, z)\right)$, using the associativity of $\oplus$.

(b) If $\inf \left(1_{\mathcal{L}^{I}}, x \oplus y\right)=\left[(x \oplus y)_{1}, 1\right]$, then $\mathcal{S}_{\oplus}\left(\mathcal{S}_{\oplus}(x, y), z\right)=\inf \left(1_{\mathcal{L}^{I}},[(x \oplus\right.$ $\left.\left.y)_{1}, 1\right] \oplus z\right)$. Since $z \geq_{L^{I}} 0_{\mathcal{L}^{I}}$, we obtain that $(x \oplus(y \oplus z))_{2} \geq(x \oplus$ $\left.\left(y \oplus 0_{\mathcal{L}^{I}}\right)\right)_{2}=(x \oplus y)_{2} \geq 1$. On the other hand $\left(\left[(x \oplus y)_{1}, 1\right] \oplus z\right)_{2} \geq$ $\left(\left[(x \oplus y)_{1}, 1\right] \oplus 0_{\mathcal{L}^{I}}\right)_{2}=1$. So $\left(\mathcal{S}_{\oplus}\left(\mathcal{S}_{\oplus}(x, y), z\right)\right)_{2}=\left(\mathcal{S}_{\oplus}\left(x, \mathcal{S}_{\oplus}(y, z)\right)\right)_{2}$.

If $\left(\left[(x \oplus y)_{1}, 1\right] \oplus z\right)_{1} \geq 1$, then $(x \oplus(y \oplus z))_{1}=((x \oplus y) \oplus z)_{1} \geq$ $\left(\left[(x \oplus y)_{1}, 1\right] \oplus z\right)_{1} \geq 1$. If $\left(\left[(x \oplus y)_{1}, 1\right] \oplus z\right)_{1}<1$, then from (6) it follows that $\left(\mathcal{S}_{\oplus}\left(\mathcal{S}_{\oplus}(x, y), z\right)\right)_{1}=\left(\mathcal{S}_{\oplus}\left(x, \mathcal{S}_{\oplus}(y, z)\right)\right)_{1}$.

(c) If $\inf \left(1_{\mathcal{L}^{I}}, x \oplus y\right)=1_{\mathcal{L}^{I}}$, then $\mathcal{S}_{\oplus}\left(\mathcal{S}_{\oplus}(x, y), z\right)=\inf \left(1_{\mathcal{L}^{I}}, 1_{\mathcal{L}^{I}} \oplus z\right)=$ $1_{\mathcal{L}^{I}}$. On the other hand, $x \oplus(y \oplus z) \geq_{\mathcal{L}^{I}} 1_{\mathcal{L}^{I}} \oplus z \geq_{L^{I}} 1_{\mathcal{L}^{I}}$, so $\mathcal{S}_{\oplus}\left(x, \mathcal{S}_{\oplus}(y, z)\right)=1_{\mathcal{L}^{I}}$

(2) $\inf \left(1_{\mathcal{L}^{I}}, y \oplus z\right)=\left[(y \oplus z)_{1}, 1\right]$ : in this case $\mathcal{S}_{\oplus}\left(x, \mathcal{S}_{\oplus}(y, z)\right)=\inf \left(1_{\mathcal{L}^{I}}, x \oplus\right.$ $\left.\left[(y \oplus z)_{1}, 1\right]\right)$.

(a) If $\inf \left(1_{\mathcal{L}^{I}}, x \oplus y\right)=\left[(x \oplus y){ }_{1}, 1\right]$, then $\mathcal{S}_{\oplus}\left(\mathcal{S}_{\oplus}(x, y), z\right)=\inf \left(1_{\mathcal{L}^{I}},[(x \oplus\right.$ $\left.\left.y)_{1}, 1\right] \oplus z\right)$. We have that $\left(x \oplus\left[(y \oplus z)_{1}, 1\right]\right)_{2} \geq\left(\left[(y \oplus z)_{1}, 1\right]\right)_{2}=1$ and $\left(\left[(x \oplus y)_{1}, 1\right] \oplus z\right)_{2} \geq\left(\left[(x \oplus y)_{1}, 1\right]\right)_{2}=1$. So $\left(\mathcal{S}_{\oplus}\left(x, \mathcal{S}_{\oplus}(y, z)\right)\right)_{2}=$ $\left(\mathcal{S}_{\oplus}\left(\mathcal{S}_{\oplus}(x, y), z\right)\right)_{2}$.

If $\left(\left[(x \oplus y)_{1}, 1\right] \oplus z\right)_{1}<1$, then from (6) it follows that $\left(\mathcal{S}_{\oplus}\left(\mathcal{S}_{\oplus}(x, y)\right.\right.$, $z))_{1}=\left(\mathcal{S}_{\oplus}\left(x, \mathcal{S}_{\oplus}(y, z)\right)\right)_{1}$.

If on the other hand $\left(\left[(x \oplus y)_{1}, 1\right] \oplus z\right)_{1} \geq 1$, then $\left(x \oplus\left[(y \oplus z)_{1}, 1\right]\right)_{1} \geq$ 1 , otherwise from $(6)$ it would follow that $\left(x \oplus\left[(y \oplus z)_{1}, 1\right]\right)_{1}=(z \oplus$ $\left.\inf \left(1_{\mathcal{L}^{I}}, x \oplus y\right)\right)_{1}=\left(z \oplus\left[(x \oplus y)_{1}, 1\right]\right)_{1}<1$, which is a contradiction. 
(b) If $\inf \left(1_{\mathcal{L}^{I}}, x \oplus y\right)=1_{\mathcal{L}^{I}}$, then $\mathcal{S}_{\oplus}\left(\mathcal{S}_{\oplus}(x, y), z\right)=1_{\mathcal{L}^{I}}$. Since $y \oplus z \geq_{L^{I}} y$ and $y_{2} \leq 1$, we have that $\left[(y \oplus z)_{1}, 1\right] \geq_{L^{I}} y$. So we obtain $x \oplus[(y \oplus$ $\left.z)_{1}, 1\right] \geq_{L^{I}} x \oplus y \geq_{L^{I}} 1_{\mathcal{L}^{I}}$. Hence $\mathcal{S}_{\oplus}\left(x, \mathcal{S}_{\oplus}(y, z)\right)=1_{\mathcal{L}^{I}}$.

(3) $\inf \left(1_{\mathcal{L}^{I}}, y \oplus z\right)=1_{\mathcal{L}^{I}}$ : in this case $\mathcal{S}_{\oplus}\left(x, \mathcal{S}_{\oplus}(y, z)\right)=\mathcal{S}_{\oplus}\left(x, 1_{\mathcal{L}^{I}}\right)=1_{\mathcal{L}^{I}}$. Similarly, from $\inf \left(1_{\mathcal{L}^{I}}, x \oplus y\right)=1_{\mathcal{L}^{I}}$ it follows that $\mathcal{S}_{\oplus}\left(\mathcal{S}_{\oplus}(x, y), z\right)=1_{\mathcal{L}^{I}}$.

Using the commutativity of $\mathcal{S}_{\oplus}$, the other cases follow immediately from the above.

Clearly, if $x, y$ are elements of $D$, then $\mathcal{S}_{\oplus}(x, y)=\inf \left(1_{\mathcal{L}^{I}},\left[x_{1}+y_{1}, x_{1}+y_{1}\right]\right)=$ $\left[S_{W}\left(x_{1}, y_{1}\right), S_{W}\left(x_{1}, y_{1}\right)\right]$.

Theorem 16 shows that in order to check whether a mapping $\mathcal{S}_{\oplus}$ given by (5) is a t-conorm, it is sufficient to check the associativity for all $x, y, z$ in $L^{I}$ such that $\left(\inf \left(1_{\mathcal{L}^{I}}, x \oplus y\right) \oplus z\right)_{1}<1$ and $(x \oplus y)_{2}>1$.

Theorem 17 The mapping $\mathcal{T}_{\oplus}:\left(L^{I}\right)^{2} \rightarrow L^{I}$ defined by, for all $x, y$ in $L^{I}$,

$$
\mathcal{T}_{\oplus}(x, y)=\sup \left(0_{\mathcal{L}^{I}}, x \ominus\left(1_{\mathcal{L}^{I}} \ominus y\right)\right)
$$

is a t-norm on $\mathcal{L}^{I}$ if and only if $\oplus$ satisfies (6). Furthermore, $\mathcal{T}_{\oplus}$ is a natural extension of $T_{W}$ to $L^{I}$.

PROOF. Assume that $\oplus$ satisfies (6). From Theorem 16 it follows that $\mathcal{S}_{\oplus}$ is a t-conorm. Denote by $\mathcal{T}_{\oplus}$ the dual t-norm of $\mathcal{S}_{\oplus}$ w.r.t. $\mathcal{N}_{s}$. We prove that $\mathcal{T}_{\oplus}(x, y)=\sup \left(0_{\mathcal{L}^{I}}, x \ominus\left(1_{\mathcal{L}^{I}} \ominus y\right)\right)$, for all $x, y$ in $L^{I}$. First note that for any $a, b$ in $L^{I}, \mathcal{N}_{s}(\inf (a, b))=\left[1-\min \left(a_{2}, b_{2}\right), 1-\min \left(a_{1}, b_{1}\right)\right]=\left[\max \left(1-a_{2}, 1-\right.\right.$ $\left.\left.b_{2}\right), \max \left(1-a_{1}, 1-b_{1}\right)\right]=\sup \left(\mathcal{N}_{s}(a), \mathcal{N}_{s}(b)\right)$. Furthermore, for all $a \in L^{I}$, we have that $\mathcal{N}_{s}(a)=1_{\mathcal{L}^{I}} \ominus a$. Hence, we obtain, for all $x, y$ in $L^{I}$,

$$
\begin{aligned}
\mathcal{T}_{\oplus}(x, y) & =\mathcal{N}_{s}\left(\inf \left(1_{\mathcal{L}^{I}}, \mathcal{N}_{s}(x) \oplus \mathcal{N}_{s}(y)\right)\right) \\
& =\sup \left(\mathcal{N}_{s}\left(1_{\mathcal{L}^{I}}\right), \mathcal{N}_{s}\left(\mathcal{N}_{s}(x) \oplus \mathcal{N}_{s}(y)\right)\right) \\
& =\sup \left(0_{\mathcal{L}^{I}}, 1_{\mathcal{L}^{I}} \ominus\left(\left(1_{\mathcal{L}^{I}} \ominus x\right) \oplus\left(1_{\mathcal{L}^{I}} \ominus y\right)\right)\right) \\
& =\sup \left(0_{\mathcal{L}^{I}}, x \ominus\left(1_{\mathcal{L}^{I}} \ominus y\right)\right)
\end{aligned}
$$

using the definition of $\ominus$.

Conversely, assume that the mapping $\mathcal{T}_{\oplus}$ given by $(7)$ is a t-norm on $\mathcal{L}^{I}$. Then it is shown similarly that the dual t-conorm of $\mathcal{T}_{\oplus}$ w.r.t. $\mathcal{N}_{s}$ is equal to the mapping $\mathcal{S}_{\oplus}$ defined by (5). From Theorem 16 it follows that $\oplus$ satisfies (6).

If $x, y$ are elements of $D$, then from Theorem 9 it follows that $\mathcal{T}_{\oplus}(x, y)=$ $\sup \left(0_{\mathcal{L}^{I}},\left[x_{1}-\left(1-y_{1}\right), x_{1}-\left(1-y_{1}\right)\right]\right)=\left[T_{W}\left(x_{1}, y_{1}\right), T_{W}\left(x_{1}, y_{1}\right)\right]$. 
Remark 18 Note that from the proof of Theorem 17 it follows that if the mapping $\mathcal{T}_{\oplus}$ defined by (7) is a t-norm, then it is the dual t-norm of the $t$ conorm $\mathcal{S}_{\oplus}$ defined by $(5)$.

The following theorem gives a simpler sufficient condition so that $\mathcal{S}_{\oplus}$ is a t-conorm and $\mathcal{T}_{\oplus}$ is a t-norm on $\mathcal{L}^{I}$.

Theorem 19 Assume that $\oplus$ satisfies the following condition:

$$
\begin{aligned}
& \left(\forall(x, y) \in \bar{L}_{+}^{I} \times L^{I}\right) \\
& \left.\left.\left(\left(\left(\left[x_{1}, 1\right] \oplus y\right)_{1}<1 \text { and } x_{2} \in\right] 1,2\right]\right) \Longrightarrow\left(\left[x_{1}, 1\right] \oplus y\right)_{1}=(x \oplus y)_{1}\right) .
\end{aligned}
$$

Then the mappings $\mathcal{T}_{\oplus}, \mathcal{S}_{\oplus}:\left(L^{I}\right)^{2} \rightarrow L^{I}$ defined by, for all $x, y$ in $L^{I}$,

$$
\begin{aligned}
& \mathcal{T}_{\oplus}(x, y)=\sup \left(0_{\mathcal{L}^{I}}, x \ominus\left(1_{\mathcal{L}^{I}} \ominus y\right)\right), \\
& \mathcal{S}_{\oplus}(x, y)=\inf \left(1_{\mathcal{L}^{I}}, x \oplus y\right),
\end{aligned}
$$

are a $t$-norm and a $t$-conorm on $\mathcal{L}^{I}$ respectively. Furthermore $\mathcal{T}_{\oplus}$ is a natural extension of $T_{W}$ to $L^{I}$, and $\mathcal{S}_{\oplus}$ is a natural extension of $S_{W}$ to $L^{I}$.

PROOF. Note that for all $x, y$ in $L^{I}, 0_{\mathcal{L}^{I}}=0_{\mathcal{L}^{I}} \oplus 0_{\mathcal{L}^{I}} \leq_{L^{I}} x \oplus y \leq_{L^{I}} 1_{\mathcal{L}^{I}} \oplus$ $1_{\mathcal{L}^{I}}=[2,2]$, so $x \oplus y \in \bar{L}_{+}^{I}$ and $(x \oplus y)_{2} \leq 2$. From $\left(\inf \left(1_{\mathcal{L}^{I}}, x \oplus y\right) \oplus z\right)_{1}<1$ it follows that $(x \oplus y)_{1}<1$. If furthermore $(x \oplus y)_{2}>1$, then $\inf \left(1_{\mathcal{L}^{I}}, x \oplus y\right)=$ $\left[(x \oplus y)_{1}, 1\right]$. So, for any $x, y, z$ in $L^{I}$, from $\left(\inf \left(1_{\mathcal{L}^{I}}, x \oplus y\right) \oplus z\right)_{1}<1$ and $(x \oplus y)_{2}>1$ it follows, using (8), that

$$
\left(\inf \left(1_{\mathcal{L}^{I}}, x \oplus y\right) \oplus z\right)_{1}=((x \oplus y) \oplus z)_{1} .
$$

If $\inf \left(1_{\mathcal{L}^{I}}, y \oplus z\right)=y \oplus z$, then clearly $((x \oplus y) \oplus z)_{1}=\left(x \oplus \inf \left(1_{\mathcal{L}^{I}}, y \oplus z\right)\right)_{1}$. If $\inf \left(1_{\mathcal{L}^{I}}, y \oplus z\right)=\left[(y \oplus z)_{1}, 1\right]$, then $\left(x \oplus \inf \left(1_{\mathcal{L}^{I}}, y \oplus z\right)\right)_{1}<1$, because otherwise $(x \oplus(y \oplus z))_{1} \geq\left(x \oplus \inf \left(1_{\mathcal{L}^{I}}, y \oplus z\right)\right)_{1} \geq 1$, which contradicts the above. So, similarly as above it follows from (8) that $\left(x \oplus \inf \left(1_{\mathcal{L}^{I}}, y \oplus z\right)\right)_{1}=$ $(x \oplus(y \oplus z))_{1}$. The case $\inf \left(1_{\mathcal{L}^{I}}, y \oplus z\right)=1_{\mathcal{L}^{I}}$ cannot occur because otherwise $\left(\inf \left(1_{\mathcal{L}^{I}}, x \oplus y\right) \oplus z\right)_{1}=(x \oplus(y \oplus z))_{1} \geq(y \oplus z)_{1} \geq 1$, which is a contradiction. Hence in all cases $\left(\inf \left(1_{\mathcal{L}^{I}}, x \oplus y\right) \oplus z\right)_{1}=\left(x \oplus \inf \left(1_{\mathcal{L}^{I}}, y \oplus z\right)\right)_{1}$, so (6) holds. From Theorems 16 and 17 it now follows that $\mathcal{S}_{\oplus}$ and $\mathcal{T}_{\oplus}$ are a t-conorm and a t-norm on $\mathcal{L}^{I}$ respectively.

Theorem 20 The mapping $\mathcal{T}_{\otimes}:\left(L^{I}\right)^{2} \rightarrow L^{I}$ defined by, for all $x, y$ in $L^{I}$,

$$
\mathcal{T}_{\otimes}(x, y)=x \otimes y
$$

is a $t$-norm on $\mathcal{L}^{I}$. Furthermore $\mathcal{T}_{\otimes}$ is a natural extension of $T_{P}$ to $L^{I}$. 
PROOF. This follows immediately from (MUL-1)-(MUL-4).

In the following theorem an alternative way of extending the Lukasiewicz tnorm on the unit interval to $\mathcal{L}^{I}$ using the arithmetic operators on $\bar{L}^{I}$ is given.

Theorem 21 The mapping $\mathcal{T}_{\oplus}^{\prime}:\left(L^{I}\right)^{2} \rightarrow L^{I}$ defined by, for all $x, y$ in $L^{I}$,

$$
\mathcal{T}_{\oplus}^{\prime}(x, y)=\sup \left(0_{\mathcal{L}^{I}}, x \oplus\left(y \ominus 1_{\mathcal{L}^{I}}\right)\right),
$$

is a t-norm on $\mathcal{L}^{I}$ if and only if $\oplus$ satisfies the following conditions:

$$
\left(\forall a \in L^{I}\right)\left(1_{\mathcal{L}^{I}} \oplus\left(a \ominus 1_{\mathcal{L}^{I}}\right)=a\right)
$$

and

$$
\begin{aligned}
& \left(\forall(x, y, z) \in\left(L^{I}\right)^{3}\right) \\
& \left(\left(\left(\sup \left(0_{\mathcal{L}^{I}}, x \oplus\left(y \ominus 1_{\mathcal{L}^{I}}\right)\right) \oplus\left(z \ominus 1_{\mathcal{L}^{I}}\right)\right)_{2}>0 \text { and }\left(x \oplus\left(y \ominus 1_{\mathcal{L}^{I}}\right)\right)_{1}<0\right)\right. \\
& \quad \Longrightarrow\left(\sup \left(0_{\mathcal{L}^{I}}, x \oplus\left(y \ominus 1_{\mathcal{L}^{I}}\right)\right) \oplus\left(z \ominus 1_{\mathcal{L}^{I}}\right)\right)_{2} \\
& \left.\quad=\left(x \oplus\left(\sup \left(0_{\mathcal{L}^{I}}, y \oplus\left(z \ominus 1_{\mathcal{L}^{I}}\right)\right) \ominus 1_{\mathcal{L}^{I}}\right)\right)_{2}\right) .
\end{aligned}
$$

Furthermore $\mathcal{T}_{\oplus}^{\prime}$ is a natural extension of $T_{W}$ to $L^{I}$.

PROOF. Define the mapping $\mathcal{T}_{\oplus}^{\prime}:\left(L^{I}\right)^{2} \rightarrow L^{I}$ by, for all $x, y$ in $L^{I}, \mathcal{T}_{\oplus}^{\prime}(x, y)=$ $\sup \left(0_{\mathcal{L}^{I}}, x \oplus\left(y \ominus 1_{\mathcal{L}^{I}}\right)\right)$. Clearly if $\mathcal{T}_{\oplus}^{\prime}$ is a t-norm on $\mathcal{L}^{I}$, then from the associativity of $\mathcal{T}_{\oplus}^{\prime}$ it follows that $\left(\sup \left(0_{\mathcal{L}^{I}}, x \oplus\left(y \ominus 1_{\mathcal{L}^{I}}\right)\right) \oplus\left(z \ominus 1_{\mathcal{L}^{I}}\right)\right)_{2}=\left(x \oplus\left(\sup \left(0_{\mathcal{L}^{I}}, y \oplus\right.\right.\right.$ $\left.\left.\left.\left(z \ominus 1_{\mathcal{L}^{I}}\right)\right) \ominus 1_{\mathcal{L}^{I}}\right)\right)_{2}$, for all $x, y, z$ in $L^{I}$ such that $\left(\sup \left(0_{\mathcal{L}^{I}}, x \oplus\left(y \ominus 1_{\mathcal{L}^{I}}\right)\right) \oplus(z \ominus\right.$ $\left.\left.1_{\mathcal{L}^{I}}\right)\right)_{2}>0$. Hence $(11)$ holds. From the commutativity of $\mathcal{T}_{\oplus}^{\prime}$ it follows (taking into account that $\left.1_{\mathcal{L}^{I}} \oplus\left(a \ominus 1_{\mathcal{L}^{I}}\right) \geq_{L^{I}} 1_{\mathcal{L}^{I}} \oplus\left(\left[a_{1}, a_{1}\right] \ominus 1_{\mathcal{L}^{I}}\right)=\left[a_{1}, a_{1}\right] \geq_{\mathcal{L}^{I}} 0_{\mathcal{L}^{I}}\right)$ that $1_{\mathcal{L}^{I}} \oplus\left(a \ominus 1_{\mathcal{L}^{I}}\right)=\sup \left(0_{\mathcal{L}^{I}}, 1_{\mathcal{L}^{I}} \oplus\left(a \ominus 1_{\mathcal{L}^{I}}\right)\right)=\mathcal{T}_{\oplus}^{\prime}\left(1_{\mathcal{L}^{I}}, a\right)=\mathcal{T}_{\oplus}^{\prime}\left(a, 1_{\mathcal{L}^{I}}\right)=$ $\sup \left(0_{\mathcal{L}^{I}}, a \oplus\left(1_{\mathcal{L}^{I}} \ominus 1_{\mathcal{L}^{I}}\right)\right)=a$, for all $a \in L^{I}$.

Assume conversely that (10) and (11) hold. Then we prove that $\mathcal{T}_{\oplus}^{\prime}$ is a t-norm. It is easy to see that $\mathcal{T}_{\oplus}^{\prime}$ is increasing and that $\mathcal{T}_{\oplus}^{\prime}\left(1_{\mathcal{L}^{I}}, x\right)=x$, for all $x \in L^{I}$. We prove that $\mathcal{T}_{\oplus}^{\prime}$ is commutative and that $a \oplus\left(b \ominus 1_{\mathcal{L}^{I}}\right)=(a \oplus b) \oplus[-1,-1]$, for all $a, b$ in $L^{I}$. Using (10) we obtain successively, for all $a \in L^{I}$,

$$
\begin{aligned}
& 1_{\mathcal{L}^{I}} \oplus\left(a \ominus 1_{\mathcal{L}^{I}}\right)=a, \\
& {[-1,-1] \oplus 1_{\mathcal{L}^{I}} \oplus\left(a \ominus 1_{\mathcal{L}^{I}}\right)=[-1,1] \oplus a,} \\
& a \ominus 1_{\mathcal{L}^{I}}=a \oplus[-1,-1],
\end{aligned}
$$

using (ADD-1), (ADD-2), (ADD-4) and (ADD-5). It follows that $a \oplus\left(b \ominus 1_{\mathcal{L}^{I}}\right)=$ $a \oplus(b \oplus[-1,-1])=(a \oplus b) \oplus[-1,-1]$, for all $a, b$ in $L^{I}$. So, $\mathcal{T}_{\oplus}^{\prime}(x, y)=$ 
$\sup \left(0_{\mathcal{L}^{I}},(x \oplus y) \oplus[-1,-1]\right)=\mathcal{T}_{\oplus}^{\prime}(y, x)$, for all $x, y$ in $L^{I}$. The associativity is proven in a similar way as in Theorem 16.

Corollary 22 Assume that $\oplus$ satisfies (ADD-5'). The mapping $\mathcal{T}_{\oplus}^{\prime}:\left(L^{I}\right)^{2} \rightarrow$ $L^{I}$ defined by ${ }^{1}$, for all $x, y$ in $L^{I}$,

$$
\mathcal{T}_{\oplus}^{\prime}(x, y)=\sup \left(0_{\mathcal{L}^{I}}, x \oplus y \ominus 1_{\mathcal{L}^{I}}\right)
$$

is a $t$-norm on $\mathcal{L}^{I}$ if and only if $\oplus$ satisfies the following condition:

$$
\begin{aligned}
& \left(\forall(x, y, z) \in\left(L^{I}\right)^{3}\right) \\
& \left(\left(\left(\sup \left(0_{\mathcal{L}^{I}}, x \oplus y \ominus 1_{\mathcal{L}^{I}}\right) \oplus z \ominus 1_{\mathcal{L}^{I}}\right)_{2}>0 \text { and }\left(x \oplus y \ominus 1_{\mathcal{L}^{I}}\right)_{1}<0\right)\right. \\
& \left.\Longrightarrow\left(\sup \left(0_{\mathcal{L}^{I}}, x \oplus y \ominus 1_{\mathcal{L}^{I}}\right) \oplus z \ominus 1_{\mathcal{L}^{I}}\right)_{2}=\left(x \oplus \sup \left(0_{\mathcal{L}^{I}}, y \oplus z \ominus 1_{\mathcal{L}^{I}}\right) \ominus 1_{\mathcal{L}^{I}}\right)_{2}\right) .
\end{aligned}
$$

Furthermore $\mathcal{T}_{\oplus}^{\prime}$ is a natural extension of $T_{W}$ to $L^{I}$.

PROOF. From Theorem 9(xii) and (ADD-4) it follows that (10) holds. The result now easily follows from the previous theorem.

Example 23 We give t-norms $\mathcal{T}_{\oplus}, \mathcal{T}_{\otimes}$ and t-conorms $\mathcal{S}_{\oplus}$ on $\mathcal{L}^{I}$ defined using the examples for $\oplus$ and $\ominus$ given in Example 8 .

- Let $\oplus, \ominus$ and $\otimes$ be the addition, subtraction and multiplication used in the interval calculus, then, for all $x, y$ in $L^{I}$,

$$
\begin{aligned}
\mathcal{T}_{\oplus}(x, y) & =\mathcal{T}_{\oplus}^{\prime}(x, y) \\
& =\left[\max \left(0, x_{1}+y_{1}-1\right), \max \left(0, x_{2}+y_{2}-1\right)\right] \\
& =\left[T_{W}\left(x_{1}, y_{1}\right), T_{W}\left(x_{2}, y_{2}\right)\right] \\
& =\mathcal{T}_{T_{W}, T_{W}}(x, y), \\
\mathcal{T}_{\otimes}(x, y) & =\mathcal{T}_{T_{P}, T_{P}}(x, y), \\
\mathcal{S}_{\oplus}(x, y) & =\left[\min \left(1, x_{1}+y_{1}\right), \min \left(1, x_{2}+y_{2}\right)\right] \\
& =\left[S_{W}\left(x_{1}, y_{1}\right), S_{W}\left(x_{2}, y_{2}\right)\right] \\
& =\mathcal{S}_{S_{W}, S_{W}}(x, y) .
\end{aligned}
$$

Thus the t-norms $\mathcal{T}_{\oplus}, \mathcal{T}_{\oplus}^{\prime}, \mathcal{T}_{\otimes}$ and the t-conorm $\mathcal{S}_{\oplus}$ obtained using the arithmetic operators from the interval calculus are t-representable.

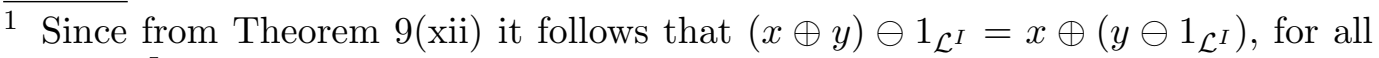
$x, y$ in $L^{I}$, we will omit the brackets in this formula. 
- Using $\oplus_{\mathcal{L}^{I}}, \ominus_{\mathcal{L}^{I}}$ and $\otimes_{\mathcal{L}^{I}}$ we obtain, for all $x, y$ in $L^{I}$,

$$
\begin{aligned}
\mathcal{T}_{\oplus_{\mathcal{L}^{I}}}(x, y) & =\left[\max \left(0, x_{1}+y_{1}-1\right), \max \left(0, x_{1}+y_{2}-1, x_{2}+y_{1}-1\right)\right] \\
& =\left[T_{W}\left(x_{1}, y_{1}\right), \max \left(T_{W}\left(x_{1}, y_{2}\right), T_{W}\left(x_{2}, y_{1}\right)\right)\right] \\
& =\mathcal{T}_{W}(x, y), \\
\mathcal{T}_{\otimes_{\mathcal{L}^{I}}}(x, y) & =\mathcal{T}_{P}(x, y), \\
\mathcal{S}_{\oplus_{\mathcal{L}^{I}}}(x, y) & =\left[\min \left(1, x_{1}+y_{2}, x_{2}+y_{1}\right), \min \left(1, x_{2}+y_{2}\right)\right] \\
& =\left[\min \left(S_{W}\left(x_{1}, y_{2}\right), S_{W}\left(x_{2}, y_{1}\right)\right), S_{W}\left(x_{2}, y_{2}\right)\right] \\
& =\mathcal{S}_{W}(x, y), \\
\mathcal{T}_{\oplus_{\mathcal{L}^{I}}}^{\prime}(x, y) & =\left[\max \left(0, \min \left(x_{1}+y_{2}-1, x_{2}+y_{1}-1\right)\right), \max \left(0, x_{2}+y_{2}-1\right)\right] \\
& =\left[\min \left(T_{W}\left(x_{1}, y_{2}\right), T_{W}\left(x_{2}, y_{1}\right)\right), T_{W}\left(x_{2}, y_{2}\right)\right] \\
& =\mathcal{T}_{T_{W}}^{\prime}(x, y) .
\end{aligned}
$$

Thus the t-norm $\mathcal{T}_{\oplus_{\mathcal{L}} I}$ and the t-conorm $\mathcal{S}_{\oplus_{\mathcal{L}} I}$ are the Eukasiewicz t-norm and t-conorm on $\mathcal{L}^{I}$, and $\mathcal{T}_{\otimes}$ is the product t-norm on $\mathcal{L}^{I}$, which are pseudot-representable. On the other hand, $\mathcal{T}_{\oplus_{\mathcal{L}^{I}}}^{\prime}$ is not pseudo-t-representable.

- Using $\oplus_{\mathcal{L}^{I}}^{t}, \ominus_{\mathcal{L}^{I}}^{t}$ and $\otimes_{\mathcal{L}^{I}}^{t}$ we obtain, for all $x, y$ in $L^{I}$,

$$
\begin{aligned}
\mathcal{T}_{\oplus_{\mathcal{L}^{I}}^{t}}(x, y)= & {\left[\max \left(0, x_{1}+y_{1}-1\right),\right.} \\
& \left.\max \left(0, t+x_{2}+y_{2}-2, x_{1}+y_{2}-1, x_{2}+y_{1}-1\right)\right] \\
= & {\left[T_{W}\left(x_{1}, y_{1}\right), \max \left(T_{W}\left(t, T_{W}\left(x_{2}, y_{2}\right)\right), T_{W}\left(x_{1}, y_{2}\right), T_{W}\left(x_{2}, y_{1}\right)\right)\right] } \\
= & \mathcal{T}_{T_{W}, t}(x, y), \\
\mathcal{T}_{\otimes_{\mathcal{L}^{I}}}(x, y)= & \mathcal{T}_{T_{P}, t}(x, y), \\
\mathcal{S}_{\oplus_{\mathcal{L}^{I}}^{t}}(x, y)= & {\left[\min \left(1,1-t+x_{1}+y_{1}, x_{1}+y_{2}, x_{2}+y_{1}\right), \min \left(1, x_{2}+y_{2}\right)\right] } \\
= & {\left[\min \left(S_{W}\left(1-t, S_{W}\left(x_{1}, y_{1}\right)\right), S_{W}\left(x_{1}, y_{2}\right), S_{W}\left(x_{2}, y_{1}\right)\right), S_{W}\left(x_{2}, y_{2}\right)\right] } \\
= & \mathcal{S}_{S_{W}, t}(x, y), \\
\mathcal{T}_{\oplus_{\mathcal{L}^{I}}^{t}}^{\prime}(x, y)= & {\left[\max \left(0, \min \left(x_{1}+y_{1}-t, x_{1}+y_{2}-1, x_{2}+y_{1}-1\right)\right),\right.} \\
& \left.\max \left(0, x_{2}+y_{2}-1\right)\right] \\
= & {\left[\operatorname { m i n } \left(\max \left(0, x_{1}+y_{1}-t\right), \max \left(0, x_{1}+y_{2}-1\right),\right.\right.} \\
& \left.\left.\max \left(0, x_{2}+y_{1}-1\right)\right), \max \left(0, x_{2}+y_{2}-1\right)\right] .
\end{aligned}
$$

- Using $\oplus_{\mathcal{L}^{I}}^{\prime}, \ominus_{\mathcal{L}^{I}}^{\prime}$ and $\otimes_{\mathcal{L}^{I}}^{\prime}$ we obtain, for all $x, y$ in $L^{I}$,

$$
\begin{aligned}
\mathcal{T}_{\oplus_{\mathcal{L}^{I}}^{\prime}}(x, y) & =\left[\max \left(0, \min \left(x_{1}+y_{2}-1, x_{2}+y_{1}-1\right)\right), \max \left(0, x_{2}+y_{2}-1\right)\right] \\
& =\left[\min \left(T_{W}\left(x_{1}, y_{2}\right), T_{W}\left(x_{2}, y_{1}\right)\right), T_{W}\left(x_{2}, y_{2}\right)\right] \\
& =\mathcal{T}_{T_{W}}^{\prime}(x, y) \\
\mathcal{T}_{\otimes_{\mathcal{L}^{I}}^{\prime}}(x, y) & =\mathcal{T}_{T_{P}}^{\prime}(x, y), \\
\mathcal{S}_{\oplus_{\mathcal{L}^{I}}^{\prime}}(x, y) & =\left[\min \left(1, x_{1}+y_{1}\right), \min \left(1, \max \left(x_{1}+y_{2}, x_{2}+y_{1}\right)\right)\right] \\
& \left.=\left[S_{W}\left(x_{1}, y_{1}\right), \max \left(S_{W}\left(x_{1}, y_{2}\right)\right), S_{W}\left(x_{2}, y_{1}\right)\right)\right] \\
& =\mathcal{S}_{S_{W}}^{\prime}(x, y),
\end{aligned}
$$




$$
\begin{aligned}
\mathcal{T}_{\oplus_{\mathcal{L}^{I}}^{\prime}}^{\prime}(x, y) & =\left[\max \left(0, x_{1}+y_{1}-1\right), \max \left(0, x_{1}+y_{2}-1, x_{2}+y_{1}-1\right)\right] \\
& =\mathcal{T}_{W}(x, y)
\end{aligned}
$$

- Using $\tilde{\oplus}_{\mathcal{L}^{I}}, \tilde{\ominus}_{\mathcal{L}^{I}}$ and $\tilde{\otimes}_{\mathcal{L}^{I}}$ we obtain, for all $x, y$ in $L^{I}$,

$$
\begin{aligned}
\mathcal{T}_{\tilde{\oplus}_{\mathcal{L}^{I}}}(x, y)= & {\left[\max \left(0, x_{1}+y_{1}-1\right),\right.} \\
& \left.\max \left(0,2 x_{1}+y_{2}-2,2 y_{1}+x_{2}-2, x_{1}+y_{1}-1\right)\right], \\
\mathcal{T}_{\tilde{\otimes}_{\mathcal{L}^{I}}}(x, y)= & {\left[x_{1} y_{1}, \max \left(\left(x_{2}+1\right) y_{1}-1,\left(y_{2}+1\right) x_{1}-1, x_{1} y_{1}\right)\right], } \\
\mathcal{S}_{\tilde{\oplus}_{\mathcal{L}^{I}}}(x, y)= & {\left[\min \left(1,2 x_{2}+y_{1}, 2 y_{2}+x_{1}, x_{2}+y_{2}\right), \min \left(1, x_{2}+y_{2}\right)\right] . }
\end{aligned}
$$

Note that $\mathcal{T}_{\tilde{\oplus}_{\mathcal{L}^{I}}}$ and $\mathcal{T}_{\tilde{\otimes}_{\mathcal{L}^{I}}}$ are the t-norms $\mathcal{T}_{1}$ and $\mathcal{T}_{2}$ given in Example 7.1 and Example 8.1 of [3], respectively.

Since $1_{\mathcal{L}^{I}} \tilde{\oplus}_{\mathcal{L}^{I}}\left([0,1] \tilde{\Theta}_{\mathcal{L}^{I}} 1_{\mathcal{L}^{I}}\right)=1_{\mathcal{L}^{I}} \tilde{\oplus}_{\mathcal{L}^{I}}[-1,-1]=[-1,0] \neq[0,1]$, we have that (10) does not hold and thus $\mathcal{T}_{\tilde{\oplus}_{\mathcal{L}^{I}}}^{\prime}$ is not a t-norm on $\mathcal{L}^{I}$.

\section{The arithmetic operators and special families of t-norms}

In this section we will generalize the Frank t-norms [13], the Schweizer-Sklar tnorms $[20,21]$ and the Yager t-norms [23] to $\mathcal{L}^{I}$ using the arithmetic operators on $\bar{L}^{I}$. In order to do so, we have to extend the exponential function and the Neperian logarithm function to $\bar{L}^{I}$.

Consider mappings $\mathfrak{e x p}: \bar{L}^{I} \rightarrow \bar{L}_{+, 0}^{I}$ and $\mathfrak{h n}: \bar{L}_{+, 0}^{I} \rightarrow \bar{L}^{I}$ which satisfy the following properties:

(EXP-1) $\mathfrak{e x p}$ is increasing,

$(\operatorname{EXP}-2) \mathfrak{e x p}([\alpha, \alpha])=\left[\mathrm{e}^{\alpha}, \mathrm{e}^{\alpha}\right]$, for all $\alpha \in \mathbb{R}$,

$(\mathrm{LN}-1) \mathfrak{l n}$ is increasing,

$(\mathrm{LN}-2) \ln ([\alpha, \alpha])=[\ln (\alpha), \ln (\alpha)]$, for all $\alpha \in] 0,+\infty[$.

In some cases we will also assume that $\mathfrak{e x p}$ and $\mathfrak{h}$ satisfy the following property:

(EXPLN) $\mathfrak{e x p}$ and $\mathfrak{h}$ are each others inverse, i.e. for all $a \in \bar{L}^{I}, \mathfrak{I n}(\mathfrak{e x p}(a))=a$, and for all $a \in \bar{L}_{+, 0}^{I}, \mathfrak{e x p}(\mathfrak{h} \mathfrak{n}(a))=a$.

Theorem 24 The mappings $\mathfrak{e x p}$ and $\mathfrak{n}$ satisfying the conditions (EXP-1), (EXP-2), (LN-1), (LN-2) and (EXPLN) are given by, for all $x \in \bar{L}^{I}$,

$$
\begin{aligned}
\mathfrak{e x p}(x) & =\left[\mathrm{e}^{x_{1}}, \mathrm{e}^{x_{2}}\right], \\
\mathfrak{l n}(x) & =\left[\ln \left(x_{1}\right), \ln \left(x_{2}\right)\right], \text { if } x_{1}>0 .
\end{aligned}
$$


PROOF. Let arbitrarily $x \in \bar{L}^{I}$ such that $x_{1}<x_{2}$. Then $x \geq_{L^{I}}\left[x_{1}, x_{1}\right]$, so $\mathfrak{e x p}(x) \geq_{L^{I}} \mathfrak{e x p}\left(\left[x_{1}, x_{1}\right]\right)$. Assume that $(\mathfrak{e x p}(x))_{1}>\left(\mathfrak{e x p}\left(\left[x_{1}, x_{1}\right]\right)\right)_{1}$. From (EXP2) it follows that $\mathfrak{e x p}\left(\left[x_{1}, x_{1}\right]\right) \in \bar{D}$. If $\mathfrak{e x p}(x)$ were an element of $\bar{D}$, then from $(\mathrm{LN}-2)$ it would follow that $\mathfrak{h}(\mathfrak{e} \mathfrak{e x p}(x))=x$ is also an element of $\bar{D}$, which is a contradiction. Hence $(\mathfrak{e x p}(x))_{1}<(\mathfrak{e x p}(x))_{2}$. Let now $z=\left[\left(\mathfrak{e x p}\left(\left[x_{1}, x_{1}\right]\right)\right)_{1}\right.$, $\left.(\mathfrak{e x p}(x))_{2}\right]$ and $z^{\prime}=\left[(\mathfrak{e x p}(x))_{1},(\mathfrak{e x p}(x))_{1}\right]$. Then from $\left(\mathfrak{e x p}\left(\left[x_{1}, x_{1}\right]\right)\right)_{1}<(\mathfrak{e x p}(x))_{1}$ $<(\mathfrak{e x p}(x))_{2}$ it follows that $z$ and $z^{\prime}$ are incomparable elements of $\bar{L}^{I}$. Furthermore, $\mathfrak{e x p}\left(\left[x_{1}, x_{1}\right]\right) \leq_{L^{I}} z \leq_{L^{I}} \mathfrak{e x p}(x)$ and $\mathfrak{e x p}\left(\left[x_{1}, x_{1}\right]\right) \leq_{L^{I}} z^{\prime} \leq_{L^{I}} \mathfrak{e x p}(x)$. Since $\mathfrak{l n}$ is increasing and is the inverse of $\mathfrak{e x p}$, we obtain that $\left[x_{1}, x_{1}\right] \leq_{L^{I}} \mathfrak{l n}(z) \leq_{L^{I}} x$ and similarly for $\mathfrak{h n}\left(z^{\prime}\right)$. But then, since $\left(\left[x_{1}, x_{1}\right]\right)_{1}=x_{1}, \mathfrak{l n}(z)$ and $\mathfrak{h n}\left(z^{\prime}\right)$ are comparable. Thus, using the fact that $\mathfrak{e x p}$ is increasing, it follows that $z$ and $z^{\prime}$ are comparable, which is a contradiction. Hence $(\mathfrak{e x p}(x))_{1}=\left(\mathfrak{e x p}\left(\left[x_{1}, x_{1}\right]\right)\right)_{1}$.

In a similar way we obtain that $(\mathfrak{e x p}(x))_{2}=\left(\mathfrak{e x p}\left(\left[x_{2}, x_{2}\right]\right)\right)_{2}$, for all $x \in \bar{L}^{I}$. From (EXP-2) it follows that $\left(\mathfrak{e x p}\left(\left[x_{1}, x_{1}\right]\right)\right)_{1}=\mathrm{e}^{x_{1}}$ and $\left(\mathfrak{e x p}\left(\left[x_{2}, x_{2}\right]\right)\right)_{2}=\mathrm{e}^{x_{2}}$, which completes the proof for $\mathfrak{e x p}$.

Let now arbitrarily $x \in \bar{L}_{+, 0}^{I}$. Using (EXPLN) and the first part of the proof, we obtain $\mathfrak{h}(x)=\mathfrak{l n}\left(\left[\mathrm{e}^{\ln \left(x_{1}\right)}, \mathrm{e}^{\ln \left(x_{2}\right)}\right]\right)=\mathfrak{l n}\left(\mathfrak{e} \mathfrak{x p}\left(\left[\ln \left(x_{1}\right), \ln \left(x_{2}\right)\right]\right)\right)=\left[\ln \left(x_{1}\right), \ln \left(x_{2}\right)\right]$.

Define for all $a, b$ in $\bar{L}_{+}^{I}$ such that $a_{1}>1$,

$$
a^{b}=\mathfrak{e x p}_{a}(b)=\mathfrak{e x p}(b \otimes \mathfrak{l n}(a)),
$$

and for all $a, b$ in $\bar{L}^{I}$ such that $a_{1}>1$ and $b_{1} \geq 1$,

$$
\mathfrak{l o g} \mathfrak{g}_{a}(b)=\mathfrak{l n}(b) \oslash \mathfrak{l n}(a)
$$

Note that the condition $a_{1}>1$ implies that $\mathfrak{h}(a) \geq_{L^{I}} \mathfrak{l n}\left(\left[a_{1}, a_{1}\right]\right)=\left[\ln \left(a_{1}\right)\right.$, $\ln \left(a_{1}\right)$ ], with $\ln \left(a_{1}\right)>0$, so we can use the operators $\otimes$ and $\oslash$ in the above formulas. Then clearly $\mathfrak{e x p}_{a}$ and $\mathfrak{l o g}_{a}$ are increasing, for all $a \in \bar{L}^{I}$ such that $a_{1}>1$.

Theorem 25 Assume that $\oplus$ and $\otimes$ satisfy (ADD-5') and (MUL-5'). Let $a \in$ $\bar{D}$ such that $a_{1}>1$. Then, $\mathfrak{e x p}_{a}$ and $\mathfrak{l o g}_{a}$ are each others inverse if and only if $\left.\mathfrak{e x p}\right|_{\bar{L}_{+}^{I}}$ and $\left.\mathfrak{l n}\right|_{\left\{x \mid x \in \bar{L}^{I} \text { and } x_{1} \geq 1\right\}}$ are each others inverse. In this case, for all $x \in \bar{L}_{+}^{I}$,

$$
\begin{aligned}
a^{x} & =\left[\left(a_{1}\right)^{x_{1}},\left(a_{1}\right)^{x_{2}}\right], \\
\log _{a}(x) & =\left[\log _{a_{1}}\left(x_{1}\right), \log _{a_{1}}\left(x_{2}\right)\right], \text { if } x_{1} \geq 1 .
\end{aligned}
$$

PROOF. Let $a \in \bar{D}$ such that $a_{1}>1$. Assume first that $\left.\mathfrak{e x p}\right|_{\bar{L}_{+}^{I}}$ and $\left.\mathfrak{l n}\right|_{\left\{x \mid x \in \bar{L}^{I} \text { and } x_{1} \geq 1\right\}}$ 
are each others inverse. Using (LN-2) and Theorem 9, we obtain, for all $x \in \bar{L}_{+}^{I}$,

$$
\begin{aligned}
\mathfrak{l o g}_{a}\left(a^{x}\right) & =\mathfrak{l n}(\mathfrak{e x p}(x \otimes \mathfrak{l n}(a))) \oslash \mathfrak{l n}(a) \\
& =(x \otimes \mathfrak{l n}(a)) \oslash \mathfrak{l n}(a) \\
& =\left[x_{1} \cdot \ln \left(a_{1}\right), x_{2} \cdot \ln \left(a_{1}\right)\right] \oslash\left[\ln \left(a_{1}\right), \ln \left(a_{1}\right)\right] \\
& =x
\end{aligned}
$$

Similarly, we obtain that $a^{\log _{a}(x)}=x$, for all $x \in \bar{L}^{I}$ such that $x_{1} \geq 1$.

Assume conversely that $\mathfrak{e x p}_{a}$ and $\mathfrak{l o g}_{a}$ are each others inverse. Using (LN-2) and Theorem 9, we obtain for all $x \in \bar{L}_{+}^{I}$,

$$
\begin{aligned}
& \mathfrak{l o g}_{a}\left(a^{x}\right)=x \\
& \Longleftrightarrow\left[\frac{(\mathfrak{n}(\mathfrak{e x p}(x \otimes \mathfrak{l n}(a))))_{1}}{\ln \left(a_{1}\right)}, \frac{(\mathfrak{l n}(\mathfrak{e} \mathfrak{x} \mathfrak{p}(x \otimes \mathfrak{l n}(a))))_{2}}{\ln \left(a_{1}\right)}\right]=x \\
& \Longleftrightarrow \mathfrak{l n}(\mathfrak{e x p}(x \otimes \mathfrak{l n}(a)))=x \otimes \mathfrak{l n}(a) .
\end{aligned}
$$

Let now arbitrarily $x \in \bar{L}_{+}^{I}$. Define $x^{\prime}=x \oslash \mathfrak{l n}(a)$. Then, since $a \in \bar{D}$ and so $\mathfrak{l n}(a) \in \bar{D}, x=x^{\prime} \otimes \mathfrak{l n}(a)$. Thus, from the above it follows that $\mathfrak{h}(\mathfrak{e x p}(x))=x$. Similarly, we obtain that $\mathfrak{e x p}(\mathfrak{l n}(x))=x$, for arbitrary $x \in \bar{L}^{I}$ such that $x_{1} \geq 1$. Hence $\left.\mathfrak{e x p}\right|_{\bar{L}_{+}^{I}}$ and $\left.\mathfrak{l n}\right|_{\left\{x \mid x \in \bar{L}^{I} \text { and } x_{1} \geq 1\right\}}$ are each others inverse.

If $\left.\mathfrak{e x p}\right|_{\bar{L}_{+}^{I}}$ and $\left.\mathfrak{l n}\right|_{\left\{x \mid x \in \bar{L}^{I} \text { and } x_{1} \geq 1\right\}}$ are each others inverse, then using Theorem 24 (note that Theorem 24 also holds for $\left.\mathfrak{e x p}\right|_{\bar{L}_{+}^{I}}$ and $\left.\mathfrak{l n}\right|_{\left\{x \mid x \in \bar{L}^{I} \text { and } x_{1} \geq 1\right\}}$ with a similar proof) we obtain, for all $x \in \bar{L}_{+}^{I}$,

$$
\begin{aligned}
a^{x} & =\mathfrak{e x p}\left(x \otimes\left[\ln \left(a_{1}\right), \ln \left(a_{1}\right)\right]\right) \\
& =\mathfrak{e x p}\left(\left[x_{1} \cdot \ln \left(a_{1}\right), x_{2} \cdot \ln \left(a_{1}\right)\right]\right) \\
& =\left[\mathrm{e}^{x_{1} \cdot \ln \left(a_{1}\right)}, \mathrm{e}^{x_{2} \cdot \ln \left(a_{1}\right)}\right] \\
& =\left[\left(a_{1}\right)^{x_{1}},\left(a_{1}\right)^{x_{2}}\right] .
\end{aligned}
$$

The formula for $\mathfrak{l o g}_{a}$ is obtained in a similar way.

The equalities $\log _{a}\left(a^{b}\right)=b$ and $a^{\log _{a}(b)}=b$ are however not valid for general $a \in \bar{L}^{I}$ satisfying $a_{1}>1$. This is shown in the next theorem.

Theorem 26 The mappings $\mathfrak{e x p}_{a}$ and $\log _{a}$ cannot be each others inverse for all $a \in \bar{L}^{I}$ such that $a_{1}>1$.

PROOF. Assume that for all $a \in \bar{L}^{I}$ such that $a_{1}>1, \mathfrak{e x p}_{a}$ and $\mathfrak{l o g}_{a}$ are each others inverse. Then also $\left.\mathfrak{e x p}\right|_{\bar{L}_{+}^{I}}=\mathfrak{e x p}_{[\mathrm{e}, \mathrm{e}]}$ and $\left.\mathfrak{l n}\right|_{\left\{x \mid x \in \bar{L}^{I} \text { and } x_{1} \geq 1\right\}}=\mathfrak{l o g} \mathfrak{g}_{[\mathrm{e}, \mathrm{e}]}$ are 
each others inverse. So we obtain, for all $a \in \bar{L}^{I}$ satisfying $a_{1}>1$,

$$
\begin{aligned}
1_{\mathcal{L}^{I}} & =\mathfrak{l o g} \mathfrak{g}_{a}\left(a^{1} \mathcal{L}^{I}\right) \\
& =\mathfrak{l n}\left(\mathfrak{e x p}\left(1_{\mathcal{L}^{I}} \otimes \mathfrak{l n}(a)\right)\right) \oslash \mathfrak{l n}(a) \\
& =\mathfrak{l n}(a) \oslash \mathfrak{l n}(a) .
\end{aligned}
$$

Let arbitrarily $x \in \bar{L}_{+, 0}^{I}$. Define $a=\mathfrak{e x p}(x)$, then $a_{1}>1$ and $x=\mathfrak{l n}(a)$, since $\left.\mathfrak{e x p}\right|_{\bar{L}_{+}^{I}}$ and $\left.\mathfrak{l n}\right|_{\left\{x \mid x \in \bar{L}^{I} \text { and } x_{1} \geq 1\right\}}$ are each others inverse. Thus, from the above it follows that $x \oslash x=1_{\mathcal{L}^{I}}$, for all $x \in \bar{L}_{+, 0}^{I}$. From (3) and (4) it follows that $\left(1_{\mathcal{L}^{I}} \oslash x\right) \otimes x=1_{\mathcal{L}^{I}}$, for all $x \in \bar{L}_{+, 0}^{I}$. This is in contradiction with Theorem 15 .

In the following subsections we will use the functions $\mathfrak{e x p}_{a}$ and $\mathfrak{l o g}_{a}$ defined above to extend some well-known classes of t-norms on $([0,1], \leq)$ to $\mathcal{L}^{I}$. From now on we assume that $\mathfrak{e x p}$ and $\mathfrak{l n}$ satisfy (EXPLN).

\subsection{The Frank t-norms}

The family $\left(T_{\lambda}^{\mathbf{F}}\right)_{\lambda \in[0,+\infty]}$ of Frank t-norms $[13]$ on $([0,1], \leq)$ is given by, for all $x_{1}, y_{1}$ in $[0,1]$,

$$
T_{\lambda}^{\mathbf{F}}\left(x_{1}, y_{1}\right)= \begin{cases}T_{M}\left(x_{1}, y_{1}\right), & \text { if } \lambda=0, \\ T_{P}\left(x_{1}, y_{1}\right), & \text { if } \lambda=1, \\ T_{W}\left(x_{1}, y_{1}\right), & \text { if } \lambda=+\infty \\ \log _{\lambda}\left(1+\frac{\left(\lambda^{x_{1}}-1\right)\left(\lambda^{y_{1}}-1\right)}{\lambda-1}\right), & \text { otherwise. }\end{cases}
$$

Let $\lambda \in \bar{D}_{+}$such that $\lambda_{1}>1$. Define the mapping $\mathcal{T}_{\lambda}^{\mathbf{F}}:\left(L^{I}\right)^{2} \rightarrow L^{I}$ by, for all $x, y$ in $L^{I}$,

$$
\mathcal{T}_{\lambda}^{\mathbf{F}}(x, y)=\log _{\lambda}\left(1_{\mathcal{L}^{I}} \oplus\left(\left(\left(\lambda^{x} \ominus 1_{\mathcal{L}^{I}}\right) \otimes\left(\lambda^{y} \ominus 1_{\mathcal{L}^{I}}\right)\right) \oslash\left(\lambda \ominus 1_{\mathcal{L}^{I}}\right)\right)\right)
$$

We show that $\mathcal{T}_{\lambda}^{\mathbf{F}}$ is a t-norm on $\mathcal{L}^{I}$. It is easy to see that $\mathcal{T}_{\lambda}^{\mathbf{F}}$ is increasing 
and commutative. Let $x \in L^{I}$, then using Theorems 9 and 25 we obtain,

$$
\begin{aligned}
\mathcal{T}_{\lambda}^{\mathbf{F}}\left(1_{\mathcal{L}^{I}}, x\right) & =\log _{\lambda}\left(1_{\mathcal{L}^{I}} \oplus\left(\left(\left(\lambda^{1} \mathcal{L}^{I} \ominus 1_{\mathcal{L}^{I}}\right) \otimes\left(\lambda^{x} \ominus 1_{\mathcal{L}^{I}}\right)\right) \oslash\left(\lambda \ominus 1_{\mathcal{L}^{I}}\right)\right)\right) \\
& =\log _{\lambda}\left(1_{\mathcal{L}^{I}} \oplus\left(\left(\left[\lambda_{1}-1, \lambda_{1}-1\right] \otimes\left(\lambda^{x} \ominus 1_{\mathcal{L}^{I}}\right)\right) \oslash\left[\lambda_{1}-1, \lambda_{1}-1\right]\right)\right) \\
& =\log _{\lambda}\left(1_{\mathcal{L}^{I}} \oplus\left(\lambda^{x} \ominus 1_{\mathcal{L}^{I}}\right)\right) \\
& =\log _{\lambda}\left(\lambda^{x}\right) \\
& =x .
\end{aligned}
$$

Note that the last equality only holds if $\mathfrak{e x p}$ and $\mathfrak{l n}$ satisfy (EXPLN). We prove the associativity. First note that for all $x, y$ in $\bar{L}_{+}^{I}$ and $\left.\alpha \in\right] 0,+\infty[$,

$$
x \otimes(y \oslash[\alpha, \alpha])=x \otimes\left(y \otimes\left[\frac{1}{\alpha}, \frac{1}{\alpha}\right]\right)=(x \otimes y) \otimes\left[\frac{1}{\alpha}, \frac{1}{\alpha}\right]=(x \otimes y) \oslash[\alpha, \alpha]
$$

Let $x, y, z$ in $L^{I}$, then, using the fact that $\mathfrak{e x p}_{\lambda}$ and $\mathfrak{l o g}_{\lambda}$ are each others inverse, we obtain

$$
\begin{aligned}
& \mathcal{T}_{\lambda}^{\mathbf{F}}\left(x, \mathcal{T}_{\lambda}^{\mathbf{F}}(y, z)\right) \\
& =\log _{\lambda}\left(1_{\mathcal{L}^{I}} \oplus\left(\left(\lambda^{x} \ominus 1_{\mathcal{L}^{I}}\right) \otimes\left(\left(\left(\lambda^{y} \ominus 1_{\mathcal{L}^{I}}\right) \otimes\left(\lambda^{z} \ominus 1_{\mathcal{L}^{I}}\right)\right) \oslash\left(\lambda \ominus 1_{\mathcal{L}^{I}}\right)\right)\right)\right. \\
& \left.\left.\quad \oslash\left(\lambda \ominus 1_{\mathcal{L}^{I}}\right)\right)\right) \\
& =\log _{\lambda}\left(1_{\mathcal{L}^{I}} \oplus\left(\left(\left(\lambda^{x} \ominus 1_{\mathcal{L}^{I}}\right) \otimes\left(\left(\lambda^{y} \ominus 1_{\mathcal{L}^{I}}\right) \otimes\left(\lambda^{z} \ominus 1_{\mathcal{L}^{I}}\right)\right)\right) \oslash\left(\lambda \ominus 1_{\mathcal{L}^{I}}\right)\right)\right. \\
& \left.\left.\oslash\left(\lambda \ominus 1_{\mathcal{L}^{I}}\right)\right)\right)
\end{aligned}
$$

which, using the associativity of $\otimes$, is symmetrical in $x, y$ and $z$. Thus $\mathcal{T}_{\lambda}^{\mathbf{F}}$ is associative.

Hence the Frank t-norms can be extended to t-norms on $\mathcal{L}^{I}$ which, using the arithmetic operators on $\bar{L}^{I}$, can be written in a similar way as their counterparts on $([0,1], \leq)$. Note also that $\mathcal{T}_{\lambda}^{\mathbf{F}}$ is a natural extension of $T_{\lambda}^{\mathbf{F}}$ to $\mathcal{L}^{I}$. 


\subsection{The Schweizer-Sklar t-norms}

First note that for all $a \in \bar{L}_{+}^{I}$ for which $a_{1}>1$, and $b \in \bar{D}_{+}$,

$$
\begin{aligned}
a^{b} & =\mathfrak{e x p}\left(\left[b_{1}, b_{1}\right] \otimes\left[\ln \left(a_{1}\right), \ln \left(a_{2}\right)\right]\right) \\
& =\mathfrak{e x p}\left(\left[b_{1} \ln \left(a_{1}\right), b_{1} \ln \left(a_{2}\right)\right]\right) \\
& =\left[\exp \left(b_{1} \ln \left(a_{1}\right)\right), \exp \left(b_{1} \ln \left(a_{2}\right)\right)\right] \\
& =\left[\left(a_{1}\right)^{b_{1}},\left(a_{2}\right)^{b_{1}}\right] .
\end{aligned}
$$

Thus, we extend the operation $a^{b}$ to elements $a \in \bar{L}_{+}^{I}$ (including the elements for which $a_{1} \leq 1$ ) as follows: for all $a \in \bar{L}_{+}^{I}$ and $b \in \bar{D}_{+}$,

$$
a^{b}=\left[\left(a_{1}\right)^{b_{1}},\left(a_{2}\right)^{b_{1}}\right] .
$$

The family $\left(T_{\lambda}^{\mathbf{S S}}\right)_{\lambda \in[-\infty,+\infty]}$ of Schweizer-Sklar t-norms [20,21] is given by, for all $x_{1}, y_{1}$ in $[0,1]$,

$$
T_{\lambda}^{\mathbf{S S}}\left(x_{1}, y_{1}\right)= \begin{cases}T_{M}\left(x_{1}, y_{1}\right), & \text { if } \lambda=-\infty \\ T_{P}\left(x_{1}, y_{1}\right), & \text { if } \lambda=0, \\ T_{D}\left(x_{1}, y_{1}\right), & \text { if } \lambda=+\infty \\ \left(\max \left(0, x_{1}^{\lambda}+y_{1}^{\lambda}-1\right)\right)^{\frac{1}{\lambda}}, & \text { otherwise }\end{cases}
$$

Let $\lambda \in \bar{D}_{+}$. Define the mapping $\mathcal{T}_{\lambda}^{\text {SS }}:\left(L^{I}\right)^{2} \rightarrow L^{I}$ by, for all $x, y$ in $L^{I}$,

$$
\mathcal{T}_{\lambda}^{\mathrm{SS}}(x, y)=\left(\sup \left(0_{\mathcal{L}^{I}}, x^{\lambda} \ominus\left(1_{\mathcal{L}^{I}} \ominus y^{\lambda}\right)\right)\right)^{1_{\mathcal{L}^{I}} \oslash \lambda}
$$

We show that $\mathcal{T}_{\lambda}^{\text {SS }}$ is a t-norm on $\mathcal{L}^{I}$. It is easy to see that $\mathcal{T}_{\lambda}^{\text {SS }}$ is increasing. From Theorem $9(\mathrm{vi})$ it follows that $\mathcal{T}_{\lambda}^{\mathrm{SS}}$ is commutative. Note that for all $a \in \bar{L}_{+}^{I}$ and $b \in \bar{D}_{+}$,

$$
\left(a^{b}\right)^{1} \mathcal{L}^{I} \oslash b=\left[\left(a_{1}\right)^{b_{1}},\left(a_{2}\right)^{b_{1}}\right]^{\left[\frac{1}{b_{1}}, \frac{1}{b_{1}}\right]}=\left[\left(\left(a_{1}\right)^{b_{1}}\right)^{\frac{1}{b_{1}}},\left(\left(a_{2}\right)^{b_{1}}\right)^{\frac{1}{b_{1}}}\right]=a .
$$

So we obtain for all $x \in L^{I}$,

$$
\begin{aligned}
\mathcal{T}_{\lambda}^{\mathrm{SS}}\left(1_{\mathcal{L}^{I}}, x\right) & =\left(\sup \left(0_{\mathcal{L}^{I}}, 1_{\mathcal{L}^{I}} \ominus\left(1_{\mathcal{L}^{I}} \ominus x^{\lambda}\right)\right)\right){ }^{1}{ }_{\mathcal{L}^{I}} \oslash \lambda \\
& =\left(\sup \left(0_{\mathcal{L}^{I}}, 1_{\mathcal{L}^{I}} \ominus\left[1-\left(x_{2}\right)^{\lambda_{1}}, 1-\left(x_{1}\right)^{\lambda_{1}}\right]\right)\right)^{1_{\mathcal{L}^{I}} \oslash \lambda} \\
& =\left(\sup \left(0_{\mathcal{L}^{I}}, x^{\lambda}\right)\right)^{1_{\mathcal{L}^{I}} \oslash \lambda} \\
& =x
\end{aligned}
$$


We prove the associativity: for all $x, y, z$ in $L^{I}$,

$$
\begin{aligned}
& \mathcal{T}_{\lambda}^{\mathrm{SS}}\left(x, \mathcal{T}_{\lambda}^{\mathrm{SS}}(y, z)\right) \\
& \left.=\left(\sup \left(0_{\mathcal{L}^{I}}, x^{\lambda} \ominus\left(1_{\mathcal{L}^{I}} \ominus\left(\left(y^{\lambda} \ominus\left(1_{\mathcal{L}^{I}} \ominus z^{\lambda}\right)\right)\right)^{1_{\mathcal{L}^{I}} \oslash \lambda}\right){ }^{\lambda}\right)\right)\right)^{1_{\mathcal{L}^{I}} \oslash \lambda} \\
& =\left(\sup \left(0_{\mathcal{L}^{I}}, x^{\lambda} \ominus\left(1_{\mathcal{L}^{I}} \ominus\left(y^{\lambda} \ominus\left(1_{\mathcal{L}^{I}} \ominus z^{\lambda}\right)\right)\right)\right)\right)^{1_{\mathcal{L}^{I}} \oslash \lambda} \\
& =\left(\sup \left(0_{\mathcal{L}^{I}},\left(y^{\lambda} \ominus\left(1_{\mathcal{L}^{I}} \ominus z^{\lambda}\right)\right) \ominus\left(1_{\mathcal{L}^{I}} \ominus x^{\lambda}\right)\right)\right)^{1_{\mathcal{L}^{I}} \oslash \lambda} \\
& =\left(\sup \left(0_{\mathcal{L}^{I}},\left(y^{\lambda} \ominus\left(1_{\mathcal{L}^{I}} \ominus x^{\lambda}\right)\right) \ominus\left(1_{\mathcal{L}^{I}} \ominus z^{\lambda}\right)\right)\right)^{{ }_{\mathcal{L}^{I}} \oslash \lambda} \\
& =\mathcal{T}_{\lambda}^{\operatorname{SS}}\left(z, \mathcal{T}_{\lambda} \mathbf{S S}(y, x)\right) \\
& =\mathcal{T}_{\lambda}^{\mathbf{S S}}\left(\mathcal{T}_{\lambda}^{\mathbf{S S}}(x, y), z\right) .
\end{aligned}
$$

Hence the Schweizer-Sklar t-norms can be extended to t-norms on $\mathcal{L}^{I}$ which, using the arithmetic operators on $\bar{L}^{I}$, can be written in a similar way as their counterparts on $([0,1], \leq)$. Note also that $\mathcal{T}_{\lambda}^{\text {SS }}$ is a natural extension of $T_{\lambda}^{\text {SS }}$ to $\mathcal{L}^{I}$.

\subsection{The Yager t-norms}

The family $\left(T_{\lambda}^{\mathbf{Y}}\right)_{\lambda \in[0,+\infty]}$ of Yager t-norms [23] is given by, for all $x_{1}, y_{1}$ in $[0,1]$,

$$
T_{\lambda}^{\mathbf{Y}}\left(x_{1}, y_{1}\right)= \begin{cases}T_{D}\left(x_{1}, y_{1}\right), & \text { if } \lambda=0 \\ T_{M}\left(x_{1}, y_{1}\right), & \text { if } \lambda=+\infty \\ \max \left(0,1-\left(\left(1-x_{1}\right)^{\lambda}+\left(1-y_{1}\right)^{\lambda}\right)^{\frac{1}{\lambda}}\right), & \text { otherwise }\end{cases}
$$

Let $\lambda \in \bar{D}_{+}$. Define the mapping $\mathcal{T}_{\lambda}^{\mathbf{Y}}:\left(L^{I}\right)^{2} \rightarrow L^{I}$ by, for all $x, y$ in $L^{I}$,

$$
\mathcal{T}_{\lambda}^{\mathbf{Y}}(x, y)=\sup \left(0_{\mathcal{L}^{I}}, 1_{\mathcal{L}^{I}} \ominus\left(\left(1_{\mathcal{L}^{I}} \ominus x\right)^{\lambda} \oplus\left(1_{\mathcal{L}^{I}} \ominus y\right)^{\lambda}\right)^{1_{\mathcal{L}^{I}} \oslash \lambda}\right)
$$

We show that $\mathcal{T}_{\lambda}^{\mathbf{Y}}$ is a t-norm on $\mathcal{L}^{I}$. It is easy to see that $\mathcal{T}_{\lambda}^{\mathbf{Y}}$ is increasing and commutative. For all $x \in L^{I}$, we obtain

$$
\begin{aligned}
\mathcal{T}_{\lambda}^{\mathbf{Y}}\left(1_{\mathcal{L}^{I}}, x\right) & =\sup \left(0_{\mathcal{L}^{I}}, 1_{\mathcal{L}^{I}} \ominus\left(\left(1_{\mathcal{L}^{I}} \ominus x\right)^{\lambda}\right){ }^{1_{\mathcal{L}^{I}} \oslash \lambda}\right) \\
& =\sup \left(0_{\mathcal{L}^{I}}, 1_{\mathcal{L}^{I}} \ominus\left(1_{\mathcal{L}^{I}} \ominus x\right)\right) \\
& =x .
\end{aligned}
$$


We prove the associativity. Using (13), we obtain for all $x, y, z$ in $L^{I}$,

$$
\begin{aligned}
& \mathcal{T}_{\lambda}^{\mathbf{Y}}\left(x, \mathcal{T}_{\lambda}^{\mathbf{Y}}(y, z)\right) \\
& =\sup \left(0_{\mathcal{L}^{I}}, 1_{\mathcal{L}^{I}} \ominus\left(\left(1_{\mathcal{L}^{I}} \ominus x\right)^{\lambda}\right.\right. \\
& \left.\left.\oplus\left(1_{\mathcal{L}^{I}} \ominus\left(1_{\mathcal{L}^{I}} \ominus\left(\left(1_{\mathcal{L}^{I}} \ominus y\right)^{\lambda} \oplus\left(1_{\mathcal{L}^{I}} \ominus z\right)^{\lambda}\right)^{1_{\mathcal{L}^{I}} \oslash \lambda}\right)\right)^{\lambda}\right)^{1_{\mathcal{L}^{I}} \oslash \lambda}\right)
\end{aligned}
$$

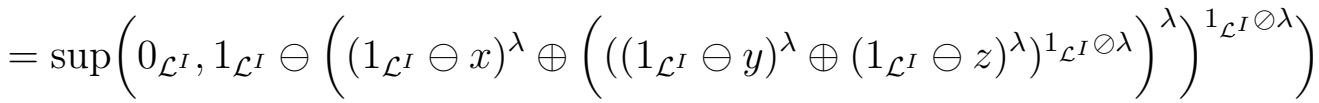

$$
\begin{aligned}
& =\sup \left(0_{\mathcal{L}^{I}}, 1_{\mathcal{L}^{I}} \ominus\left(\left(1_{\mathcal{L}^{I}} \ominus x\right)^{\lambda} \oplus\left(\left(1_{\mathcal{L}^{I}} \ominus y\right)^{\lambda} \oplus\left(1_{\mathcal{L}^{I}} \ominus z\right)^{\lambda}\right)\right)^{1_{\mathcal{L}^{I}} \oslash \lambda}\right),
\end{aligned}
$$

which is symmetrical in $x, y$ and $z$. Thus $\mathcal{T}_{\lambda}^{\mathbf{Y}}$ is associative. Hence the Yager tnorms can be extended to t-norms on $\mathcal{L}^{I}$ which, using the arithmetic operators on $\bar{L}^{I}$, can be written in a similar way as their counterparts on $([0,1], \leq)$. Note also that $\mathcal{T}_{\lambda}^{\mathbf{Y}}$ is a natural extension of $T_{\lambda}^{\mathbf{Y}}$ to $\mathcal{L}^{I}$.

Example 27 We give the expressions for the Frank, Schweizer-Sklar and Yager t-norms on $\mathcal{L}^{I}$ obtained using the examples for $\oplus, \ominus, \otimes$ and $\oslash$ given in the previous section.

- Let $\oplus, \ominus, \otimes$ and $\oslash$ be the arithmetic operators used in the interval calculus, then, for all $x, y$ in $L^{I}$,

$$
\begin{aligned}
\mathcal{T}_{\lambda}^{\mathbf{F}}(x, y)= & \log _{\lambda}\left([ 1 , 1 ] \oplus \left(\left(\left[\lambda_{1}^{x_{1}}-1, \lambda_{1}^{x_{2}}-1\right] \otimes\left[\lambda_{1}^{y_{1}}-1, \lambda_{1}^{y_{2}}-1\right]\right)\right.\right. \\
& \left.\left.\oslash\left[\lambda_{1}-1, \lambda_{1}-1\right]\right)\right) \\
= & \log _{\lambda}\left([ 1 , 1 ] \oplus \left(\left[\left(\lambda_{1}^{x_{1}}-1\right)\left(\lambda_{1}^{y_{1}}-1\right),\left(\lambda_{1}^{x_{2}}-1\right)\left(\lambda_{1}^{y_{2}}-1\right)\right]\right.\right. \\
& \left.\left.\oslash\left[\lambda_{1}-1, \lambda_{1}-1\right]\right)\right) \\
= & \log _{\lambda}\left(\left[1+\frac{\left(\lambda_{1}^{x_{1}}-1\right)\left(\lambda_{1}^{y_{1}}-1\right)}{\lambda_{1}-1}, 1+\frac{\left(\lambda_{1}^{x_{2}}-1\right)\left(\lambda_{1}^{y_{2}}-1\right)}{\lambda_{1}-1}\right]\right) \\
= & \mathcal{T}_{T_{\lambda_{1}}^{\mathrm{F}}, T_{\lambda_{1}}^{\mathrm{F}}}(x, y),
\end{aligned}
$$

and similarly

$$
\begin{aligned}
\mathcal{T}_{\lambda}^{\mathbf{S S}}(x, y) & =\mathcal{T}_{T_{\lambda_{1}}^{\mathrm{SS}}, T_{\lambda_{1}}^{\mathrm{SS}}}(x, y), \\
\mathcal{T}_{\lambda}^{\mathbf{Y}}(x, y) & =\mathcal{T}_{T_{\lambda_{1}} \mathbf{Y}, T_{\lambda_{1}}}^{\mathbf{Y}}(x, y) .
\end{aligned}
$$

Thus the t-norms $\mathcal{T}_{\lambda}^{\mathbf{F}}, \mathcal{T}_{\lambda}^{\text {SS }}$ and $\mathcal{T}_{\lambda}^{\mathbf{Y}}$ obtained using the arithmetic operators from the interval calculus are t-representable.

Note that, for all $x, y$ in $L^{I}$,

$$
\begin{aligned}
\lim _{\lambda \rightarrow 1_{\mathcal{L}^{I}}, \lambda \in \bar{D}_{+}} \mathcal{T}_{\lambda}^{\mathbf{F}}(x, y) & =\left[\lim _{\lambda_{1} \rightarrow 1} T_{\lambda_{1}}^{\mathbf{F}}\left(x_{1}, y_{1}\right), \lim _{\lambda_{1} \rightarrow 1} T_{\lambda_{1}}^{\mathbf{F}}\left(x_{2}, y_{2}\right)\right] \\
& =\left[T_{P}\left(x_{1}, y_{1}\right), T_{P}\left(x_{2}, y_{2}\right)\right] \\
& =\mathcal{T}_{T_{P}, T_{P}}(x, y) .
\end{aligned}
$$


Here we calculated the limit in the metric space $\left(L^{I}, d^{E}\right),\left(L^{I}, d^{M}\right)$ or any other topologically equivalent metric space, where the Euclidean metric $d^{E}$ and the max-metric $d^{H}$ are given by

$$
\begin{aligned}
d^{E}\left(\left[x_{1}, x_{2}\right],\left[y_{1}, y_{2}\right]\right) & =\sqrt{\left(x_{1}-y_{1}\right)^{2}+\left(x_{2}-y_{2}\right)^{2}}, \\
d^{M}\left(\left[x_{1}, x_{2}\right],\left[y_{1}, y_{2}\right]\right) & =\max \left(\left|x_{1}-y_{1}\right|,\left|x_{2}-y_{2}\right|\right),
\end{aligned}
$$

for all $\left[x_{1}, x_{2}\right],\left[y_{1}, y_{2}\right]$ in $L^{I}$.

Similarly we obtain

$$
\begin{array}{rlrl}
\lim _{\lambda \rightarrow[+\infty,+\infty], \lambda \in \bar{D}_{+}} \mathcal{T}_{\lambda}^{\mathbf{F}}(x, y) & =\mathcal{T}_{T_{W}, T_{W}}(x, y), \\
\lim _{\lambda \rightarrow 0} \mathcal{T}_{\mathcal{L}^{I}, \lambda \in \bar{D}_{+}} \mathcal{T}_{\lambda}^{\mathbf{S S}}(x, y) & =\mathcal{T}_{T_{P}, T_{P}}(x, y), & \lim _{\lambda \rightarrow[+\infty,+\infty], \lambda \in \bar{D}_{+}} & \mathcal{T}_{\lambda}^{\mathbf{S S}}(x, y)=\mathcal{T}_{T_{D}, T_{D}}(x, y), \\
\lim _{\lambda \rightarrow 0} \mathcal{L}_{\mathcal{L}^{I}, \lambda \in \bar{D}_{+}} & \mathcal{T}_{\lambda}^{\mathbf{Y}}(x, y)=\mathcal{T}_{T_{D}, T_{D}}(x, y), & \lim _{\lambda \rightarrow[+\infty,+\infty], \lambda \in \bar{D}_{+}} & \mathcal{T}_{\lambda}^{\mathbf{Y}}(x, y)=\mathcal{T}_{T_{M}, T_{M}}(x, y) .
\end{array}
$$

- Using $\oplus_{\mathcal{L}^{I}}, \ominus_{\mathcal{L}^{I}}, \otimes_{\mathcal{L}^{I}}$ and $\oslash_{\mathcal{L}^{I}}$ we obtain, for all $x, y$ in $L^{I}$,

$$
\begin{aligned}
\mathcal{T}_{\lambda}^{\mathbf{F}}(x, y)= & \log _{\lambda}\left([ 1 , 1 ] \oplus _ { \mathcal { L } ^ { I } } \left(\left(\left[\lambda_{1}^{x_{1}}-1, \lambda_{1}^{x_{2}}-1\right] \otimes_{\mathcal{L}^{I}}\left[\lambda_{1}^{y_{1}}-1, \lambda_{1}^{y_{2}}-1\right]\right)\right.\right. \\
& \left.\left.\oslash_{\mathcal{L}^{I}}\left[\lambda_{1}-1, \lambda_{1}-1\right]\right)\right) \\
= & \log _{\lambda}\left([ 1 , 1 ] \oplus _ { \mathcal { L } ^ { I } } \left(\left[\left(\lambda_{1}^{x_{1}}-1\right)\left(\lambda_{1}^{y_{1}}-1\right),\right.\right.\right. \\
& \left.\max \left(\left(\lambda_{1}^{x_{1}}-1\right)\left(\lambda_{1}^{y_{2}}-1\right),\left(\lambda_{1}^{x_{2}}-1\right)\left(\lambda_{1}^{y_{1}}-1\right)\right)\right] \\
& \left.\left.\oslash_{\mathcal{L}^{I}}\left[\lambda_{1}-1, \lambda_{1}-1\right]\right)\right) \\
= & \log _{\lambda}\left([ 1 , 1 ] \oplus _ { \mathcal { L } ^ { I } } \left[\frac{\left(\lambda_{1}^{x_{1}}-1\right)\left(\lambda_{1}^{y_{1}}-1\right)}{\lambda_{1}-1},\right.\right. \\
& \left.\left.\quad \max \left(\frac{\left(\lambda_{1}^{x_{1}}-1\right)\left(\lambda_{1}^{y_{2}}-1\right)}{\lambda_{1}-1}, \frac{\left(\lambda_{1}^{x_{2}}-1\right)\left(\lambda_{1}^{y_{1}}-1\right)}{\lambda_{1}-1}\right)\right]\right) \\
= & {\left[\log _{\lambda}\left(1+\frac{\left(\lambda_{1}^{x_{1}}-1\right)\left(\lambda_{1}^{y_{1}}-1\right)}{\lambda_{1}-1}\right)\right.} \\
& \quad \max ^{x_{1}}\left(\log _{\lambda}\left(1+\frac{\left(\lambda_{1}^{x_{1}}-1\right)\left(\lambda_{1}^{y_{2}}-1\right)}{\lambda_{1}-1}\right),\right. \\
& \left.\left.\quad \log _{\lambda}\left(1+\frac{\left(\lambda_{1}^{x_{2}}-1\right)\left(\lambda_{1}^{y_{1}}-1\right)}{\lambda_{1}-1}\right)\right)\right] \\
= & {\left[T_{\lambda_{1}}^{\mathbf{F}}\left(x_{1}, y_{1}\right), \max \left(T_{\lambda_{1}}^{\mathbf{F}}\left(x_{1}, y_{2}\right), T_{\lambda_{1}}^{\mathbf{F}}\left(x_{2}, y_{2}\right)\right)\right] } \\
= & \mathcal{T}_{T_{\lambda_{1}}^{\mathbf{F}}}(x, y),
\end{aligned}
$$

and similarly

$$
\begin{gathered}
\mathcal{T}_{\lambda}^{\mathbf{S S}}(x, y)=\mathcal{T}_{T_{\lambda_{1}}^{\mathbf{S S}}(x, y),} \\
\mathcal{T}_{\lambda}^{\mathbf{Y}}(x, y)=\mathcal{T}_{T_{\lambda_{1}}^{\mathbf{Y}}}(x, y) .
\end{gathered}
$$

Thus the t-norms $\mathcal{T}_{\lambda}^{\mathbf{F}}, \mathcal{T}_{\lambda}^{\mathrm{SS}}$ and $\mathcal{T}_{\lambda}^{\mathbf{Y}}$ obtained using the arithmetic operators $\oplus_{\mathcal{L}^{I}}, \ominus_{\mathcal{L}^{I}}, \otimes_{\mathcal{L}^{I}}$ and $\oslash_{\mathcal{L}^{I}}$ are pseudo-t-representable. 
Note that, for all $x, y$ in $L^{I}$,

$$
\begin{aligned}
\lim _{\lambda \rightarrow 1_{\mathcal{L}^{I}}, \lambda \in \bar{D}_{+}} \mathcal{T}_{\lambda}^{\mathbf{F}}(x, y) & =\left[\lim _{\lambda_{1} \rightarrow 1} T_{\lambda_{1}}^{\mathbf{F}}\left(x_{1}, y_{1}\right), \lim _{\lambda_{1} \rightarrow 1} \max \left(T_{\lambda_{1}}^{\mathbf{F}}\left(x_{1}, y_{2}\right), T_{\lambda_{1}}^{\mathbf{F}}\left(x_{2}, y_{1}\right)\right)\right] \\
& =\left[\lim _{\lambda_{1} \rightarrow 1} T_{\lambda_{1}}^{\mathbf{F}}\left(x_{1}, y_{1}\right), \max \left(\lim _{\lambda_{1} \rightarrow 1} T_{\lambda_{1}}^{\mathbf{F}}\left(x_{1}, y_{2}\right), \lim _{\lambda_{1} \rightarrow 1} T_{\lambda_{1}}^{\mathbf{F}}\left(x_{2}, y_{1}\right)\right)\right] \\
& =\left[T_{P}\left(x_{1}, y_{1}\right), \max \left(T_{P}\left(x_{1}, y_{2}\right), T_{P}\left(x_{2}, y_{1}\right)\right)\right] \\
& =\mathcal{T}_{T_{P}}(x, y),
\end{aligned}
$$

and similarly

$$
\begin{array}{rlrl}
\lim _{\lambda \rightarrow[+\infty,+\infty], \lambda \in \bar{D}_{+}} & \mathcal{T}_{\lambda}^{\mathbf{F}}(x, y)=\mathcal{T}_{T_{W}}(x, y)=\mathcal{T}_{W}(x, y), & \\
\lim _{\lambda \rightarrow 0} \mathcal{L}_{\mathcal{L}^{I}, \lambda \in \bar{D}_{+}} \mathcal{T}_{\lambda}^{\mathbf{S S}}(x, y) & =\mathcal{T}_{T_{P}}(x, y), & \lim _{\lambda \rightarrow[+\infty,+\infty], \lambda \in \bar{D}_{+}} \mathcal{T}_{\lambda}^{\mathbf{S S}}(x, y)=\mathcal{T}_{T_{D}}(x, y), \\
\lim _{\lambda \rightarrow 0}{ }_{\mathcal{L}^{I}, \lambda \in \bar{D}_{+}} & \mathcal{T}_{\lambda}^{\mathbf{Y}}(x, y) & =\mathcal{T}_{T_{D}}(x, y), & \lim _{\lambda \rightarrow[+\infty,+\infty], \lambda \in \bar{D}_{+}} \mathcal{T}_{\lambda}^{\mathbf{Y}}(x, y)=\mathcal{T}_{T_{M}}(x, y) .
\end{array}
$$

- Using $\oplus_{\mathcal{L}^{I}}^{t}, \ominus_{\mathcal{L}^{I}}^{t}, \otimes_{\mathcal{L}^{I}}^{t}$ and $\oslash_{\mathcal{L}^{I}}^{t}$ we obtain, for all $x, y$ in $L^{I}$,

$$
\begin{aligned}
\mathcal{T}_{\lambda}^{\mathbf{F}}(x, y)= & \log _{\lambda}\left([ 1 , 1 ] \oplus _ { \mathcal { L } ^ { I } } ^ { t } \left(\left(\left[\lambda_{1}^{x_{1}}-1, \lambda_{1}^{x_{2}}-1\right] \otimes_{\mathcal{L}^{I}}^{t}\left[\lambda_{1}^{y_{1}}-1, \lambda_{1}^{y_{2}}-1\right]\right)\right.\right. \\
& \left.\left.\oslash_{\mathcal{L}^{I}}^{t}\left[\lambda_{1}-1, \lambda_{1}-1\right]\right)\right) \\
= & \mathfrak{l o g}_{\lambda}\left([ 1 , 1 ] \oplus _ { \mathcal { L } ^ { I } } ^ { t } \left(\left[\left(\lambda_{1}^{x_{1}}-1\right)\left(\lambda_{1}^{y_{1}}-1\right),\right.\right.\right. \\
& \left.\max \left(t\left(\lambda_{1}^{x_{2}}-1\right)\left(\lambda_{1}^{y_{2}}-1\right),\left(\lambda_{1}^{x_{1}}-1\right)\left(\lambda_{1}^{y_{2}}-1\right),\left(\lambda_{1}^{x_{2}}-1\right)\left(\lambda_{1}^{y_{1}}-1\right)\right)\right] \\
& \left.\left.\oslash_{\mathcal{L}^{I}}^{t}\left[\lambda_{1}-1, \lambda_{1}-1\right]\right)\right) \\
= & \mathfrak{l o g}_{\lambda}\left([ 1 , 1 ] \oplus _ { \mathcal { L } ^ { I } } ^ { t } \left[\frac{\left(\lambda_{1}^{x_{1}}-1\right)\left(\lambda_{1}^{y_{1}}-1\right)}{\lambda_{1}-1},\right.\right. \\
& \quad \max \left(\frac{t\left(\lambda_{1}^{x_{2}}-1\right)\left(\lambda_{1}^{y_{2}}-1\right)}{\lambda_{1}-1}, \frac{\left(\lambda_{1}^{x_{1}}-1\right)\left(\lambda_{1}^{y_{2}}-1\right)}{\lambda_{1}-1},\right. \\
& \left.\left.\left.\quad \frac{\left(\lambda_{1}^{x_{2}}-1\right)\left(\lambda_{1}^{y_{1}}-1\right)}{\lambda_{1}-1}\right)\right]\right) \\
= & {\left[\log _{\lambda}\left(1+\frac{\left(\lambda_{1}^{x_{1}}-1\right)\left(\lambda_{1}^{y_{1}}-1\right)}{\lambda_{1}-1}\right),\right.} \\
& \quad \max \left(\log _{\lambda}\left(1+\frac{\left(\lambda_{1}^{t^{\prime}}-1\right)\left(\lambda_{1}^{x_{2}}-1\right)\left(\lambda_{1}^{y_{2}}-1\right)}{\left(\lambda_{1}-1\right)^{2}}\right),\right. \\
& \left.\left.\log _{\lambda}\left(1+\frac{\left(\lambda_{1}^{x_{1}}-1\right)\left(\lambda_{1}^{y_{2}}-1\right)}{\lambda_{1}-1}\right), \log _{\lambda}\left(1+\frac{\left(\lambda_{1}^{x_{2}}-1\right)\left(\lambda_{1}^{y_{1}}-1\right)}{\lambda_{1}-1}\right)\right)\right] \\
= & {\left[T_{\lambda_{1}}^{\mathbf{F}}\left(x_{1}, y_{1}\right), \max \left(T_{\lambda_{1}}^{\mathbf{F}}\left(t^{\prime}, T_{\lambda_{1}}^{\mathbf{F}}\left(x_{2}, y_{2}\right)\right), T_{\lambda_{1}}^{\mathbf{F}}\left(x_{1}, y_{2}\right), T_{\lambda_{1}}^{\mathbf{F}}\left(x_{2}, y_{2}\right)\right)\right] } \\
= & \mathcal{T}_{T_{\lambda_{1}}^{\mathbf{F}}, t^{\prime}}(x, y), \\
&
\end{aligned}
$$

and similarly

$$
\begin{aligned}
\mathcal{T}_{\lambda}^{\mathbf{S S}}(x, y) & =\mathcal{T}_{T_{\lambda_{1}}^{\mathrm{SS}}, t}(x, y), \\
\mathcal{T}_{\lambda}^{\mathbf{Y}}(x, y) & =\mathcal{T}_{T_{\lambda_{1}}^{\mathbf{Y}}, t^{\prime \prime}}(x, y) .
\end{aligned}
$$


where $t^{\prime}$ and $t^{\prime \prime}$ are elements of $[0,1]$ which satisfy $t=\frac{\lambda_{1}^{t^{\prime}}-1}{\lambda_{1}-1}$ and $1-t=$ $\left(1-t^{\prime \prime}\right)^{\lambda_{1}}$.

Note that, similarly as for $\lim _{\lambda_{1} \rightarrow 1} T_{\lambda_{1}}^{\mathbf{F}}\left(x_{2}, y_{2}\right)=T_{P}\left(x_{2}, y_{2}\right)$, it can be proven that $\lim _{\lambda_{1} \rightarrow 1} T_{\lambda_{1}}^{\mathbf{F}}\left(t^{\prime}, T_{\lambda_{1}}^{\mathbf{F}}\left(x_{2}, y_{2}\right)\right)=T_{P}\left(t^{\prime}, T_{P}\left(x_{2}, y_{2}\right)\right)$, for all $x_{2}, y_{2}$ in $[0,1]$. So we obtain, for all $x, y$ in $L^{I}$,

$$
\begin{aligned}
& \lim _{\lambda \rightarrow 1} \mathcal{L}_{\mathcal{L}^{I}}, \lambda \in \bar{D}_{+} \\
& \mathcal{T}_{\lambda}^{\mathbf{F}}(x, y)= {\left[\lim _{\lambda_{1} \rightarrow 1} T_{\lambda_{1}}^{\mathbf{F}}\left(x_{1}, y_{1}\right), \max \left(\lim _{\lambda_{1} \rightarrow 1} T_{\lambda_{1}}^{\mathbf{F}}\left(t^{\prime}, T_{\lambda_{1}}^{\mathbf{F}}\left(x_{2}, y_{2}\right)\right),\right.\right.} \\
&\left.\left.\lim _{\lambda_{1} \rightarrow 1} T_{\lambda_{1}}^{\mathbf{F}}\left(x_{1}, y_{2}\right), \lim _{\lambda_{1} \rightarrow 1} T_{\lambda_{1}}^{\mathbf{F}}\left(x_{2}, y_{1}\right)\right)\right] \\
&= {\left[T_{P}\left(x_{1}, y_{1}\right), \max \left(T_{P}\left(t^{\prime}, T_{P}\left(x_{2}, y_{2}\right)\right), T_{P}\left(x_{1}, y_{2}\right), T_{P}\left(x_{2}, y_{1}\right)\right)\right] } \\
&= \mathcal{T}_{T_{P}, t^{\prime}}(x, y),
\end{aligned}
$$

and similarly

$$
\begin{aligned}
& \lim _{\lambda \rightarrow[+\infty,+\infty], \lambda \in \bar{D}_{+}} \mathcal{T}_{\lambda}^{\mathbf{F}}(x, y)=\mathcal{T}_{T_{W}, t^{\prime}}(x, y), \\
& \lim _{\lambda \rightarrow 0} \mathcal{L}_{\mathcal{L}^{I}, \lambda \in \bar{D}_{+}} \mathcal{T}_{\lambda}^{\mathbf{S S}}(x, y)=\mathcal{T}_{T_{P}, t}(x, y), \quad \lim _{\lambda \rightarrow[+\infty,+\infty], \lambda \in \bar{D}_{+}} \mathcal{T}_{\lambda}^{\mathbf{S S}}(x, y)=\mathcal{T}_{T_{D}, t}(x, y), \\
& \lim _{\lambda \rightarrow 0} \mathcal{T}_{\mathcal{L}^{I}, \lambda \in \bar{D}_{+}} \mathcal{T}_{\lambda}^{\mathbf{Y}}(x, y)=\mathcal{T}_{T_{D}, t^{\prime \prime}}(x, y), \quad \lim _{\lambda \rightarrow[+\infty,+\infty], \lambda \in \bar{D}_{+}} \mathcal{T}_{\lambda}^{\mathbf{Y}}(x, y)=\mathcal{T}_{T_{M}, t^{\prime \prime}}(x, y) .
\end{aligned}
$$

- Using $\oplus_{\mathcal{L}^{I}}^{\prime}, \ominus_{\mathcal{L}^{I}}^{\prime}, \otimes_{\mathcal{L}^{I}}^{\prime}$ and $\oslash_{\mathcal{L}^{I}}^{\prime}$ we obtain, for all $x, y$ in $L^{I}$,

$$
\begin{aligned}
\mathcal{T}_{\lambda}^{\mathbf{F}}(x, y) & =\mathcal{T}_{T_{\lambda_{1}}^{\mathbf{F}}}^{\prime}(x, y), \\
\mathcal{T}_{\lambda}^{\mathbf{S S}}(x, y) & =\mathcal{T}_{T_{\lambda_{1}}^{\mathbf{S S}}}^{\prime}(x, y), \\
\mathcal{T}_{\lambda}^{\mathbf{Y}}(x, y) & =\mathcal{T}_{T_{\lambda_{1}}^{\mathbf{Y}}}^{\prime}(x, y) .
\end{aligned}
$$

Note that, for all $x, y$ in $L^{I}$,

$$
\begin{array}{rrrl}
\lim _{\lambda \rightarrow 1} \mathcal{L}_{\mathcal{L}^{I}, \lambda \in \bar{D}_{+}} & \mathcal{T}_{\lambda}^{\mathbf{F}}(x, y)=\mathcal{T}_{T_{P}}^{\prime}(x, y), & \lim _{\lambda \rightarrow[+\infty,+\infty], \lambda \in \bar{D}_{+}} \mathcal{T}_{\lambda}^{\mathbf{F}}(x, y)=\mathcal{T}_{T_{W}}^{\prime}(x, y), \\
\lim _{\lambda \rightarrow 0} \mathcal{L}_{\mathcal{L}^{I}, \lambda \in \bar{D}_{+}} \mathcal{T}_{\lambda}^{\mathbf{S S}}(x, y)=\mathcal{T}_{T_{P}}^{\prime}(x, y), & \lim _{\lambda \rightarrow[+\infty,+\infty], \lambda \in \bar{D}_{+}} \mathcal{T}_{\lambda}^{\mathbf{S S}}(x, y)=\mathcal{T}_{T_{D}}^{\prime}(x, y), \\
\lim _{\lambda \rightarrow 0_{\mathcal{L}^{I}}, \lambda \in \bar{D}_{+}} \mathcal{T}_{\lambda}^{\mathbf{Y}}(x, y)=\mathcal{T}_{T_{D}}^{\prime}(x, y), & \lim _{\lambda \rightarrow[+\infty,+\infty], \lambda \in \bar{D}_{+}} \mathcal{T}_{\lambda}^{\mathbf{Y}}(x, y)=\mathcal{T}_{T_{M}}^{\prime}(x, y) .
\end{array}
$$

\section{Conclusion}

In this paper we have studied extensions of the arithmetic operators,,$+- \cdot$, $\div$ to the lattice $\mathcal{L}^{I}$ of closed subintervals of the unit interval. Starting from a minimal set of axioms that these operators must fulfill, we investigated which properties they satisfy. We have shown that the addition and multiplication operators on $\mathcal{L}^{I}$ never generate a group. Using any choice of the arithmetic operators on $\mathcal{L}^{I}$, some operators can be defined which are constructed using the 
same arithmetic formula as the Eukasiewicz t-norm and t-conorm, the product t-norm, the Frank t-norms, the Schweizer-Sklar t-norms and the Yager tnorms on the unit interval. We have given necessary and sufficient conditions such that these operators are t-norms and t-conorms on $\mathcal{L}^{I}$ and we have shown that they are natural extensions of their counterparts on the unit interval. For the Eukasiewicz t-norm, we have given two possible extensions to $\mathcal{L}^{I}$ using the arithmetic operators on $\mathcal{L}^{I}$. In order to extend the Frank, Schweizer-Sklar and Yager t-norms, we have introduced generalizations of the exponential and the logarithm functions to $\mathcal{L}^{I}$ and we have investigated some of their properties. In a future work we will construct additive and multiplicative generators of t-norms on $\mathcal{L}^{I}$ based on the arithmetic operators introduced in this paper.

\section{References}

[1] K. T. Atanassov, Intuitionistic fuzzy sets, Physica-Verlag, Heidelberg, New York, 1999.

[2] C. Cornelis, G. Deschrijver, E. E. Kerre, Implication in intuitionistic and interval-valued fuzzy set theory: construction, classification and application, International Journal of Approximate Reasoning 35 (1) (2004) 55-95.

[3] G. Deschrijver, Characterizations of (weakly) Archimedean t-norms in intervalvalued fuzzy set theory, Fuzzy Sets and SystemsIn press.

[4] G. Deschrijver, The Eukasiewicz t-norm in interval-valued fuzzy and intuitionistic fuzzy set theory, in: K. T. Atanassov, J. Kacprzyk, M. Krawczak, E. Szmidt (eds.), Issues in the Representation and Processing of Uncertain and Imprecise Information. Fuzzy Sets, Intuitionistic Fuzzy Sets, Generalized Nets, and Related Topics, Akademicka Oficyna Wydawnicza EXIT, 2005, pp. 83-101.

[5] G. Deschrijver, Additive and multiplicative generators in interval-valued fuzzy set theory, IEEE Transactions on Fuzzy Systems 15 (2) (2007) 222-237.

[6] G. Deschrijver, Arithmetic operators in interval-valued fuzzy set theory, Information Sciences 177 (14) (2007) 2906-2924.

[7] G. Deschrijver, C. Cornelis, E. E. Kerre, On the representation of intuitionistic fuzzy t-norms and t-conorms, IEEE Transactions on Fuzzy Systems 12 (1) (2004) 45-61.

[8] G. Deschrijver, E. E. Kerre, Classes of intuitionistic fuzzy t-norms satisfying the residuation principle, International Journal of Uncertainty, Fuzziness and Knowledge-Based Systems 11 (6) (2003) 691-709.

[9] G. Deschrijver, E. E. Kerre, On the relationship between some extensions of fuzzy set theory, Fuzzy Sets and Systems 133 (2) (2003) 227-235. 
[10] G. Deschrijver, E. E. Kerre, Implicators based on binary aggregation operators in interval-valued fuzzy set theory, Fuzzy Sets and Systems 153 (2) (2005) 229248.

[11] G. Deschrijver, A. Vroman, Generalized arithmetic operations in interval-valued fuzzy set theory, Journal of Intelligent and Fuzzy Systems 16 (4) (2005) 265-271.

[12] F. Esteva, P. Garcia-Calvés, L. Godo, Enriched interval bilattices and partial many-valued logics: an approach to deal with graded truth and imprecision, International Journal of Uncertainty, Fuzziness and Knowledge-Based Systems 2 (1) (1994) 37-54.

[13] M. J. Frank, On the simultaneous associativity of $f(x, y)$ and $x+y-f(x, y)$, Aequationes Math. 19 (1979) 194-226.

[14] J. A. Goguen, L-fuzzy sets, Journal of Mathematical Analysis and Applications 18 (1) (1967) 145-174.

[15] M. B. Gorzałczany, A method of inference in approximate reasoning based on interval-valued fuzzy sets, Fuzzy Sets and Systems 21 (1) (1987) 1-17.

[16] E. P. Klement, R. Mesiar, E. Pap, Triangular norms, Kluwer Academic Publishers, Dordrecht, 2000.

[17] E. P. Klement, R. Mesiar, E. Pap, Triangular norms. Position paper III: continuous t-norms, Fuzzy Sets and Systems 145 (3) (2004) 439-454.

[18] R. E. Moore, Interval arithmetic, Prentice-Hall, Englewood Cliffs, NJ, USA, 1966.

[19] R. Sambuc, Fonctions $\Phi$-floues. Application à l'aide au diagnostic en pathologie thyroidienne, Ph.D. thesis, Université de Marseille, France (1975).

[20] B. Schweizer, A. Sklar, Associative functions and abstract semigroups, Publ. Math. Debrecen 10 (1963) 69-81.

[21] B. Schweizer, A. Sklar, Probabilistic metric spaces, Elsevier, North-Holland, New York, 1983.

[22] B. Van Gasse, C. Cornelis, G. Deschrijver, E. E. Kerre, A characterization of interval-valued residuated lattices, International Journal of Approximate Reasoning 49 (2) (2008) 478-487.

[23] R. R. Yager, On a general class of fuzzy connectives, Fuzzy Sets and Systems 4 (3) (1980) 235-242. 